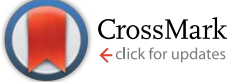

Cite this: RSC Adv., 2017, 7, 16801

\title{
Graphene, hexagonal boron nitride, and their heterostructures: properties and applications
}

Received 7th January 2017 Accepted 23rd February 2017

DOI: $10.1039 / c 7 r a 00260 b$

rsc.li/rsc-advances

\author{
Jingang Wang, ${ }^{\text {abcd }}$ Fengcai $\mathrm{Ma}^{\star \mathrm{b}}$ and Mengtao Sun ${ }^{\star a b c}$
}

In recent years, two-dimensional atomic-level thickness crystal materials have attracted widespread interest such as graphene, hexagonal boron nitride ( $h-B N)$, silicene, germanium, black phosphorus (BP), transition metal sulfides and so on. These graphene-like two-dimensional (2D) materials have a lot of excellent characteristics such as high specific surface area and high Young's modulus, and many potential applications in diverse areas such as photo-electricity, catalysts, and transistors. In this review, we introduced the synthesis, structure, properties, and applications of graphene, $\mathrm{h}-\mathrm{BN}$, and their heterostructures, especially focused on their mechanical, optical, thermal, electric, and magnetic properties. Finally, we present the outlooks and perspectives for these types of excellent 2D materials and their potential applications.

\section{Introduction to the two- dimensional materials}

\subsection{Introduction to graphene}

Graphene is a single layer of graphite sheet, constituting the basic unit of graphite, carbon nanotubes, fullerenes, and other carbon materials (Fig. 1(a)). ${ }^{1,2}$ Before the experimental discovery

aBeijing Key Laboratory for Magneto-Photoelectrical Composite and Interface Science, School of Mathematics and Physics, University of Science and Technology Beijing, Beijing, 100083, People's Republic of China. E-mail: mengtaosun@ustb.edu.cn

${ }^{b}$ Department of Chemistry and Physics, Liaoning University, Shenyang, 110036, People's Republic of China. E-mail: mafengcai@lnu.edu.cn

'Beijing National Laboratory for Condensed Matter Physics, Beijing Key Laboratory for Nanomaterials and Nanodevices, Institute of Physics, Chinese Academy of Sciences, Beijing, 100190, People's Republic of China. E-mail: mtsun@iphy.ac.cn

${ }^{d}$ Department of Physics, Shenyang Aerospace University, 110036, People's Republic of China

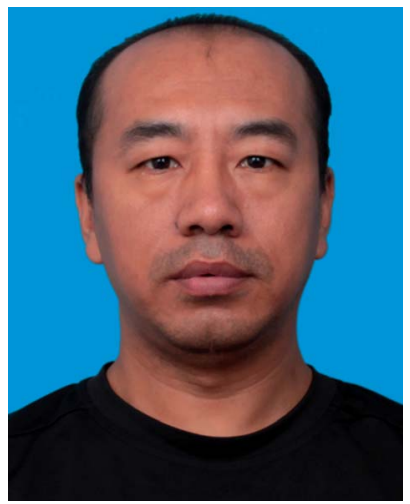

Jingang Wang is a Ph.D. candidate supervised by Prof. Fengcai Ma and Prof. Mengtao Sun at the Department of Chemistry and Physics, Liaoning University, China. His current research interests are the properties and applications of two dimensional (2D) materials. of graphene, because of the effects of thermal expansion, theoretical and experimental scientists believed that at finite temperatures strict two-dimensional crystals could not be stable. However, in 2004, Geim and Novoselov ${ }^{3}$ produced a single layer of carbon in atoms-level thickness using micromechanical exfoliation (Microexfoliation) and studied the electric field effect (see Fig. 1(c)) and carried out a series of studies on the produced graphene, which dispelled the previous hypothesis. ${ }^{4-7}$

Graphene comprises honeycomb-dimensional crystals closely arranged by $\mathrm{sp}^{2}$ hybridized carbon atoms, and hexagonal geometry makes graphene structure very stable. ${ }^{8}$ Each interlayer carbon atom bonds with the surrounding carbon atoms by $\mathrm{sp}^{2}$ hybridization, and contributes a non-bonding electron to form a large $\pi$ bond, to allow the electrons to move freely between the layers.

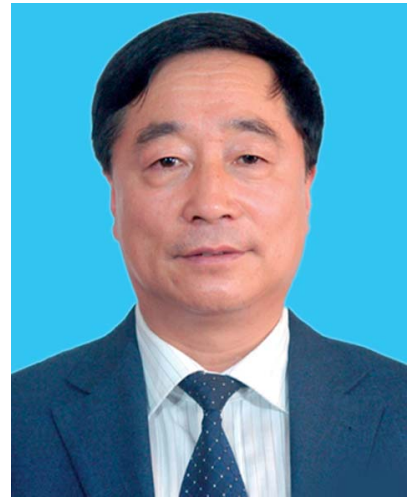

Fengcai Ma is a Professor at Liaoning University. He obtained his Ph.D. in 1991 from Lanzhou University. Since 1996, he has worked as a Professor at the Department of Physics, Liaoning University, China. His current research interests focus on the mechanism of photoinduced electron transfer dynamics in two dimensional (2D) and complex systems. 


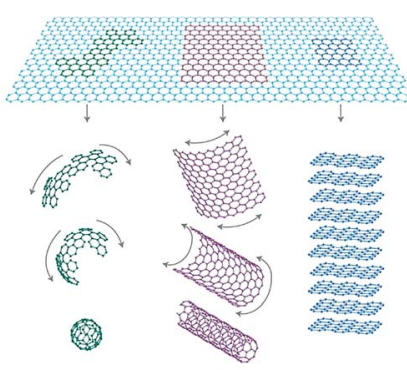

(a)

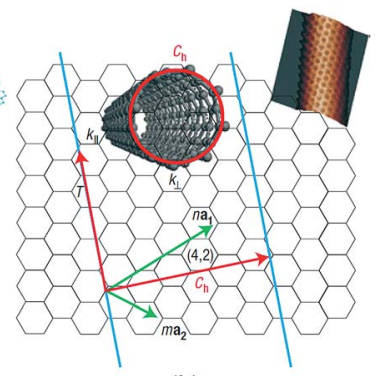

(b)

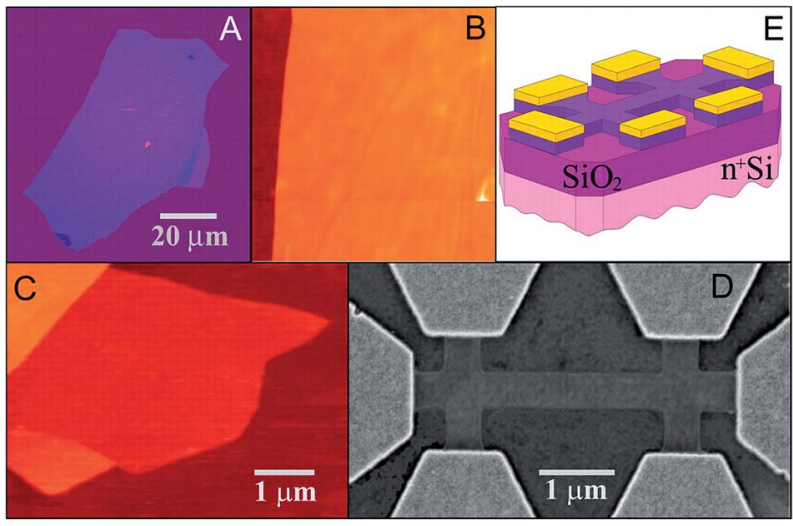

(c)

Fig. 1 (a) The basic unit of the other $C$ material - graphene. ${ }^{1}$ (b) The relational graph between graphene and carbon nanotubes. ${ }^{7}$ (c) Graphene film and devices (flow chart of graphene prepared by mechanical stripping method from $\mathrm{A}$ to $\mathrm{E}){ }^{3}$

Graphene is the thinnest and hardest nanomaterial, ${ }^{9}$ with a tensile strength of $125 \mathrm{GPa}$, an elastic modulus of $1.1 \mathrm{TPa}$, and a two-dimensional ultimate plane strength of $42 \mathrm{~N} \mathrm{~m}^{-2}$. Graphene's carrier mobility is $2 \times 10^{5} \mathrm{~cm}^{2}\left(\mathrm{~V}^{-1} \mathrm{~s}^{-1}\right)^{10,11}$ and is only affected by impurities and defects. Graphene's thermal

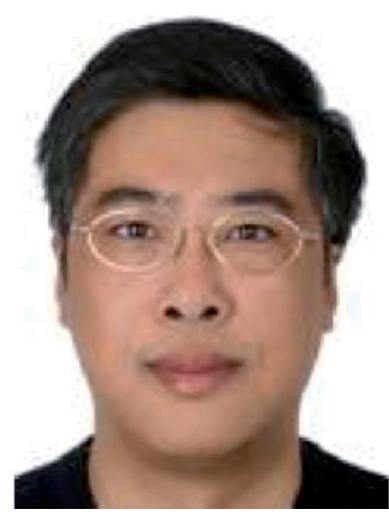

Mengtao Sun obtained his Ph.D. in 2003 from the State Key Laboratory of Molecular Reaction Dynamics, Dalian Institute of Chemical Physics, Chinese Academy of Sciences (CAS). From 2003 to 2006, he worked as a postdoc at the Department of Chemical Physics, Lund University. Since 2006, as an associate professor, he has worked at the Beijing National Laboratory for Condensed Matter Physics, Institute of Physics, CAS. In 2016, he became a Full Professor at Beijing Key Laboratory for Magneto-Photoelectrical Composite and Interface Science, the University of Science and Technology Beijing, China. His current research interests focus on two dimensional (2D) materials and plasmonics, as well as the exciton-plasmon coupling interaction of $2 D$ materials-plasmonic metal hybrids. conductivity is up to $5.5 \times 10^{3} \mathrm{~W}\left(\mathrm{~m}^{-1} \mathrm{~K}^{-1}\right){ }^{12,13}$ Theoretically, graphene's specific surface area is up to $2630 \mathrm{~m}^{2} \mathrm{~g}^{-1}$. These unique physical properties make graphene to be widely applied in many areas of nanoelectronic devices, spin electronics, energy storage, and thermal conductivity materials.

1.1.1 Carbon nanotubes (deformation of graphene): properties compared with graphene. Carbon nanotubes (CNTs) are one-dimensional nanomaterials with a special structure. ${ }^{7,14}$ It can be regarded as a nanometer-scale hollow tubular structure made of a single-layer or multi-layer graphene sheet (Fig. 1(b)). According to the different number of layers in CNTs, CNTs can be divided into SWNTs ${ }^{15-17}$ and MWNTs. ${ }^{18}$ According to the arrangement of carbon atoms in the cross-section of single wall CNTs, these can be further categorized into armchair or zigzag CNTs. ${ }^{19,20}$ According to the different electronic structures of SWNTs, these can be categorized into metallic and semiconductive (i.e. non-integral) SWNTs. ${ }^{21,22}$

The $\mathrm{C}=\mathrm{C}$ covalent bond of carbon nanotubes makes the axial Young's modulus of carbon nanotubes reach as high as 5 $\mathrm{TPa},{ }^{20,23,24}$ while the length-diameter ratio is as high as $10^{4}$, the comparison area is more than $1500 \mathrm{~m}^{2} \mathrm{~g}^{-1}$ (ref. 25 and 26) and the current carrying capacity is up to $10^{9} \mathrm{~A} \mathrm{~cm}^{-2} ;{ }^{27,28}$ One of the optical properties of carbon nanotubes is their wide band absorption. ${ }^{29,30}$ As a good thermal conductor, the axial thermal conductivity of CNTs is up to $6600 \mathrm{~W}\left(\mathrm{M}^{-1} \mathrm{~K}^{-1}\right)$, with excellent field emission characteristics, where its emission current mainly comes from the occupied states being slightly lower than the Fermi level.

As representatives of one-dimensional and two-dimensional nanomaterials, while graphene is composed only by a single carbon atomic layer, this is the true sense of a the twodimensional crystal structure. Compared with graphene, carbon nanotubes have a higher total amount of carbon atoms, making themselves have a lower energy of the edge dangling bonds than graphene, which can stabilize the molecules in the air without them reacting with the air. From a performance standpoint, graphene has similar or more excellent characteristics than carbon nanotubes in terms of conductivity, carrier mobility, thermal conductivity, free electron moving space, strength and stiffness.

Graphene and carbon-nanotubes have different applications for many reasons, but ultimately the differences can be attributed to the difference between one-dimensional materials and twodimensional material. For example, a single carbon nanotube can be regarded as a single crystal with a high length-diameter ratio; however, the current synthesis and assembly technology cannot prepare the carbon nanotube crystals in a macroscopic scale, which limits their applications. While, an advantage of graphene is that it is a two-dimensional crystal structure, and its strength, conductivity and thermal conductivity are among the best of other two-dimensional crystal materials and it has a broad range of application prospects because of the ability of having a large area of continuous growth.

\subsection{Introduction to graphene-like two-dimensional crystals - hexagonal boron nitride}

Hexagonal boron nitride (h-BN), ${ }^{31}$ which belongs to a hexagonal system, is a white block or powder, has a layered structure 


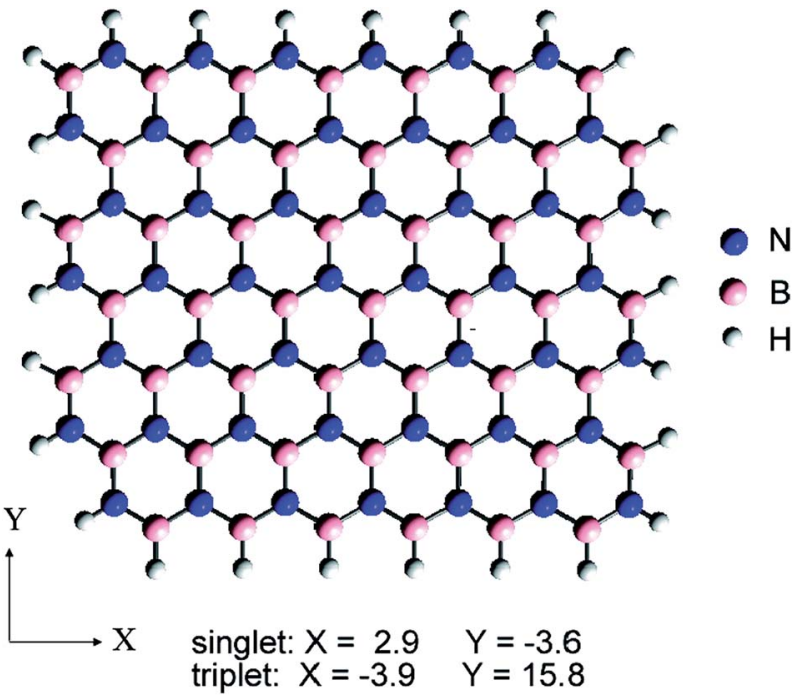

Fig. 2 Flat structure diagram of $\mathrm{h}-\mathrm{BN}$. Blue on behalf of nitrogen atoms and pink on behalf of boron atoms, respectively. ${ }^{34}$

similar to the graphene lattice constant and similar characteristics, and is sometimes referred to as 'white graphene. ${ }^{32-34} \mathrm{~h}$ $\mathrm{BN}$ is a lattice alternately arranged by $\mathrm{B}$ atoms and $\mathrm{N}$ atoms in a two-dimensional plane by hexagonal lattice formation, showing a honeycomb structure (Fig. 2). The $\mathrm{N}$ atomic nucleus and $\mathrm{B}$ atom are combined by an $\mathrm{sp}^{2}$ orbital to form a strong $\sigma$ bond, ${ }^{35-39}$ with the interlayer combined by weak van der Waals forces such that it can slide easily between the layers and has soft lubricating properties.

In 1995, Nagashima et al. used an epitaxial growth on a variety of metal surfaces to obtain $\mathrm{h}-\mathrm{BN}$ crystals. ${ }^{40} \mathrm{~A}$ team from the University of Manchester adopted a micro-mechanical peeling method in 2005 to successfully prepare a twodimensional h-BN. ${ }^{41}$ The band gap of h-BN is about $5.9 \mathrm{eV}$, while the Mohs' scale of hardness is about 2, the bulk modulus about $36.5 \mathrm{GP}$, the heat conductivity layer up to $600-1000 \mathrm{~W}$ $\mathrm{m}^{-1} \mathrm{~K}^{-1}$, the coefficient of thermal expansion layer about -2.7 $\times 10^{-6}$ per ${ }^{\circ} \mathrm{C}$ (interlayer about $30 \times 10^{-6}$ per ${ }^{\circ} \mathrm{C}$ ) and the refractive index about 1.8, and furthermore, it has neutron absorption ability. ${ }^{42-45}$ In addition, its anti-oxidation temperature is $900{ }^{\circ} \mathrm{C}$ and its thermos ability is up to $2000{ }^{\circ} \mathrm{C}$, while at $2700^{\circ} \mathrm{C}$, in an inert environment it remains stable. Finally, h-BN also has good process ability, such as thermal shock resistance to electrical vibration, high resistance to break down the electric field strength, and is non-toxic and environmentally friendly, with no wettability to various metals or chemical corrosion and has other excellent physical and chemical properties, and consequently is widely used..$^{46-53}$

\subsection{Introduction of graphene/h-BN as a two-dimensional composite}

Graphene has very good electronic properties. However, it will be a challenging to make graphene into nanoelectronic devices. Moreover, good mechanical stability is required when using scanning probe technology to detect micro-graphene. ${ }^{54} \mathrm{~A}$ substrate can be introduced to solve this problem, whereby, the introduction of the substrate can open up the graphene band gap, thereby improving the switching performance of graphene electronics.

There have already been a lot studies into graphene and the substrate materials, such as $\mathrm{Co}^{, 55} \mathrm{Ni}^{56-59} \mathrm{Ru}^{60,61}$ and $\mathrm{Pt},{ }^{62,63}$ as well as semiconductors $\mathrm{SiO}_{2}$ (ref. 64-66) and $\mathrm{SiC},{ }^{67-69}$ which can be used as a substrate graphene. However, experiments show that the graphene on top of these substrates is very uneven, and it will have a lot of wrinkles, which could restrain the properties of graphene. For example, $\mathrm{SiO}_{2}$ is the most common graphene substrate, but on the $\mathrm{SiO}_{2}$ surface there are usually impurities, which can cause the scattering of charge or act as charge traps. Therefore, the growth and charge density distribution of graphene on the $\mathrm{SiO}_{2}$ substrate is very uneven (Fig. 3), ${ }^{70}$ which results in significant suppression of the carrier mobility of graphene. Recently, a lot of research studies have proven that hexagonal boron nitride is an ideal substrate and is ideally suited for graphene to maintain its geometrical and electrical properties.

Monolayer graphene and h-BN have a similar lattice structure, with a lattice mismatch of only about $1.5 \%{ }^{71}$ As a base, h-BN has a smooth surface without any charge traps, and it also has a low dielectric constant and high temperature stability as well as high thermal conductivity and other properties. With a dielectric constant $\varepsilon$ of 3-4 and a breakdown electric field strength of about $0.7 \mathrm{~V} \mathrm{~nm}^{-1}, \mathrm{~h}-\mathrm{BN}$ is a great gate insulating layer for graphene. The surface optical phonon energy of h-BN is two orders of magnitude greater than that of $\mathrm{SiO}_{2}$, which indicates that using $\mathrm{h}$-BN as the substrate is likely to improve the performance of graphene devices in conditions such as at higher temperature and in a higher electric field. In the h-BN single cell, the difference in grid energy between nitrogen atoms and boron atoms leads to a broad band gap of about $5.9 \mathrm{eV},{ }^{72}$ which can help open the band gap of graphene. All the above show that h-BN is an ideal substrate material for graphene.
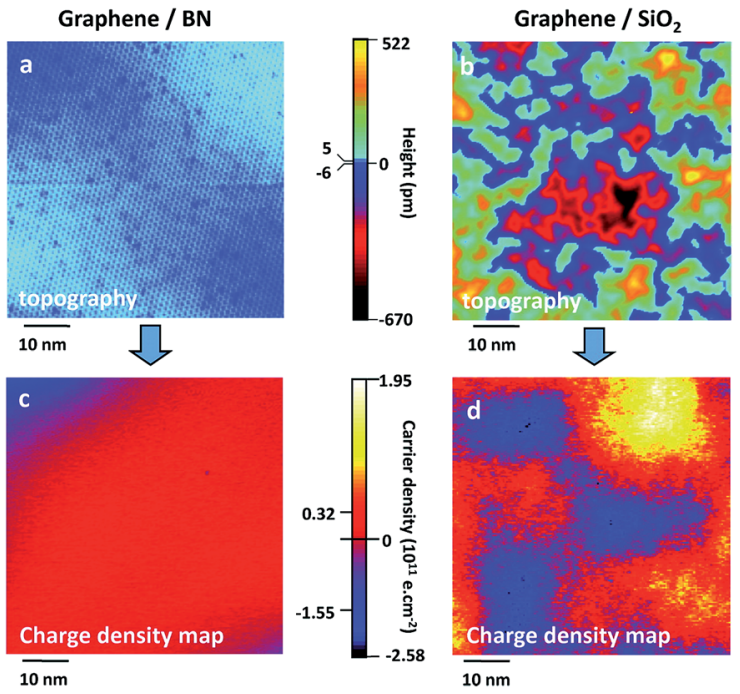

Fig. 3 Comparison of the topography and charge density for graphene/BN vs. graphene/ $/ \mathrm{SiO}_{2}{ }^{73}$ 
Dean et al., for the first time using h-BN as a supporting substrate of graphene, fabricated graphene transistors device with high mobility, ${ }^{72}$ on which a clear graphene quantum Hall effect was observed. Ponomarenko et al. (Fig. 3) (33 $^{73}$ used physical transfer to combine thin sheets of graphene and h-BN crystals to produce a heterojunction device with two single layers of graphene crystals. At the same time, researchers have succeeded in achieving a variety of graphene heterojunctions and superlattice structures with more complex structure; ${ }^{74-76}$ for instance, researchers in the UK and the US observed the novel Hofstadter Butterfly phenomenon on graphene/h-BN heterojunction devices, respectively. ${ }^{77,78}$ Since then, researchers have used a variety of methods to fabricate graphene/h-BN heterostructure functional devices, with the original purpose of improving the quality and speed of preparation of graphene instead of actually studying the performance of the graphene/hBN heterostructures.

The single layer graphene and h-BN have similar lattice structure, and lattice mismatch is only about $1.5 \% .{ }^{63}$ As a base, h-BN has smooth surface without charge trap, and it also has a low dielectric constant, high temperature stability as well as high thermal conductivity and other properties. Hexagonal boron nitride with dielectric constant $\varepsilon$ of $3-4$, breakdown electric field strength of about $0.7 \mathrm{~V} \mathrm{~nm}^{-1}$, is great gate insulating layer for graphene. The surface optical phonon energy of h-BN is two orders of magnitude greater than that of $\mathrm{SiO}_{2}$, which indicates that the using of hexagonal boron nitride as the substrate is likely to improve the performance of graphene device in conditions like high temperature and high electric field. ${ }^{17}$ In the h-BN single cell the difference of grid energy between nitrogen atoms and boron atoms leads to a broad band gap of about $5.9 \mathrm{eV},{ }^{64}$ which can help open the band gap of graphene. All above shows that $\mathrm{h}-\mathrm{BN}$ is an ideal substrate material for graphene.

\section{Graphene}

\subsection{Structure of graphene}

Ideal graphene is a single layer of two-dimensional atomic crystals with an orthohexagonal lattice structure. The length of the $\mathrm{C}-\mathrm{C}$ bond is around $0.142 \mathrm{~nm}$, and the thickness of the layer is $0.35 \mathrm{~nm}$. In each state, a single carbon atom forms a strong $\sigma$ bond with its three nearest neighbours, respectively, by $\mathrm{sp}^{2}$ orbital hybridization, causing occupied and unoccupied states to move away from each other. Each unit cell of graphene has two types of sub-lattice, type A and B (Fig. 4a), and there are chiral characteristics between the spins of electrons of $\mathrm{A}$ and B. ${ }^{79}$

\subsection{Preparation of graphene}

The preparation of graphene can be classified into physical and chemical methods, including mechanical peeling ${ }^{1}$ and epitaxial growth ${ }^{\mathbf{8 0}}$ chemical cleavage, ${ }^{\mathbf{8 1}}$ chemical vapour deposition (CVD), ${ }^{11}$ low thermal expansion, ${ }^{82}$ nanotubes cutting, ${ }^{83}$ metal catalysis and so on, ${ }^{\mathbf{8 4}, 85}$ as shown in Fig. 5.
Yu et al. summarized the progress on the direct growth of graphene on semiconductor and insulator substrates in recent years. ${ }^{86}$ Table 1 shows a comparison of the physical properties between several materials and h-BN as substrates.

\subsection{Physical properties of graphene}

2.3.1 Mechanical and thermal characteristics of graphene. The 2D honeycomb-shaped crystal structure endows graphene with excellent in-plane mechanical properties. Lee et al. ${ }^{9}$ found that the Young's modulus of graphene can reach $130 \pm 10 \mathrm{GPa}$ under the assumption of the thickness of the graphene layer of $0.35 \mathrm{~nm},{ }^{9}$ as shown in Fig. 6, and two-dimensional ultimate plane strength of $42 \mathrm{~N} \mathrm{~m}^{-2}$. Gómez-Navarro et al. ${ }^{87}$ and Poot et al. obtained the intensity values of different graphene layers by various methods. ${ }^{88}$ As shown in the above studies, graphene as a novel nanomaterial has excellent mechanical properties.

Because of the high modulus of elasticity and the long mean free path of electrons, the thermal conductivity of graphene can reach up to $3000-6000 \mathrm{~W} \mathrm{~m}^{-1} \mathrm{~K}^{-1}$. Hong et al. ${ }^{11}$ showed that graphene's thermal conductivity was $5300 \mathrm{~W} \mathrm{~m}^{-1} \mathrm{~K}^{-1}$ but decreases as the temperature increases. ${ }^{12,89}$ Seol et al. studied graphene's thermal conductivity on a $\mathrm{SiO}_{2}$ substrate $^{90}$ and the results showed that the value of thermal conductivity was still as high as $600 \mathrm{~W} \mathrm{~m}{ }^{-1} \mathrm{~K}^{-1}$, even with the phenomenon of phonon scattering occurring due to the transfer of heat between the interface of the two materials. In addition, defects and the unordered arrangement of the edges will reduce the thermal conductivity of graphene (Fig. 7). ${ }^{91}$

2.3.2 Optical properties of graphene. Single-layer graphene is colourless, so we always observe graphene with a substrate. The light absorption intensity of graphene is irrelevant to the frequency of light but appears to have a linear relationship with the amount of graphene layers (Fig. 8). ${ }^{\mathbf{9 2 9 3}}$ For a single layer of graphene, the absorption and transmittance of visible light are $2.3 \%$ and $97.7 \%$, respectively. ${ }^{94,95}$ Graphene has characteristics of a wide band absorption and zero energy gap, so in the irradiation of near-infrared powerful light, the absorption of light by graphene gets saturated and it exhibits nonlinear optical behaviour. The unique zero-band-gap structure of graphene makes it absorb light with no selectivity. In addition, the defects, shape and quality of graphene all have effects on its optical performance.

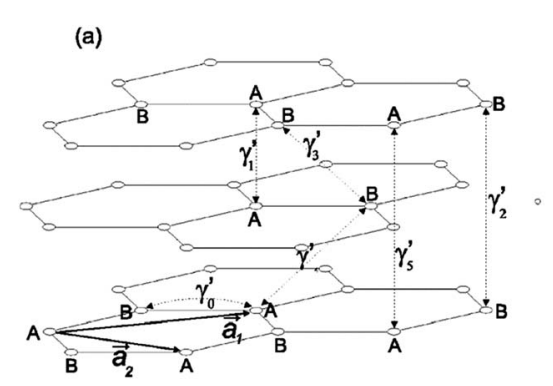

(b)

Fig. 4 (a) The relationship between distribution of $A$ and $B$ atoms in the unit cell of graphene. (b) Corresponding Brillouin zone. The Dirac cones are located at the $\mathrm{K}$ and $\mathrm{M}$ points. ${ }^{79}$ 

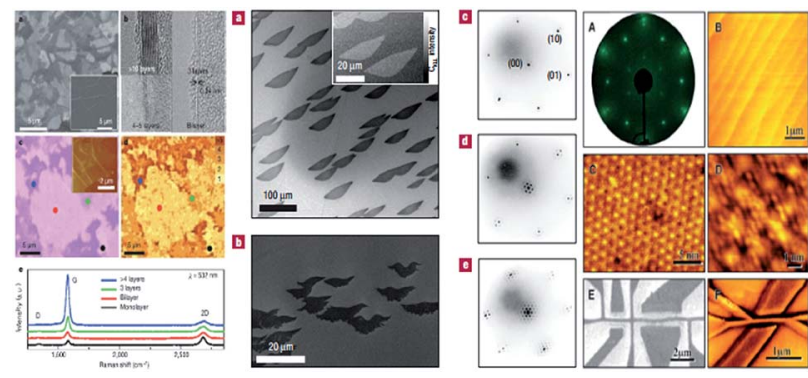

Fig. 5 Several types of preparation methods of graphene (spectral analysis of graphene prepared by CVD method is shown from a to e in the left group figures. Sample image of graphene by epitaxial growth is shown from a to e in the middle of group figures. Epitaxial growth of graphene samples and their characteristics is shown from $A$ to $E$ in the right group figures). ${ }^{11,84,85}$

2.3.3 Electrical characteristics of graphene. Graphene is a zero-band-gap semiconductor with six highly symmetrical $K$ points in the Brillouin zone, and its conduction and valence bands intersect at Dirac point (Fig. 9). ${ }^{79}$ Near the Dirac point, the energy and momentum present a linear dispersion relation $E=h v_{\mathrm{F}} K,{ }^{95}$ making the effective mass of electrons in graphene equal zero - that is, the electron in graphene is a type of massless Dirac Fermion. ${ }^{7}$

The unique band configuration of the Dirac point gives graphene excellent conductivity. Electrons in graphene pass through the barrier with $100 \%$ probability. ${ }^{96}$ Due to a strong force between the carbon atoms, the collision of carbon atoms has little effects on the motion of electrons. Chen et al., in 2008, by limitation of the external conditions found that the carrier mobility of graphene based on $\mathrm{a} \mathrm{SiO}_{2}$ substrate could reach $4 \times$ $10^{4} \mathrm{~cm}^{2} \mathrm{~V}^{-1} \mathrm{~s}^{-1},{ }^{64}$ which is comparable to the value of the best field-effect transistor, and at room temperature the carrier mobility of suspended graphene could reach up to $2 \times 10^{5} \mathrm{~cm}^{2}$ $\mathrm{V}^{-1} \mathrm{~s}^{-1}$, which is 140 times the value of silicon. Claire Biel et al. demonstrated that, in graphene, the mobility of electrons and holes are equal. ${ }^{82}$ The conductivity of graphene can reach $10^{6} \mathrm{~S}$ $\mathrm{m}^{-1}$, which means it has the best conductivity among known materials at room temperature.

Meanwhile, graphene has a bipolar field-effect ${ }^{1}$ and the transmission characteristics of a ballistic. ${ }^{82}$ Through graphene atomic doping, ${ }^{97-99}$ control of the edge structure of graphene nano-strips, ${ }^{\mathbf{1 0 0}}$ the imposing of an external electric field on the
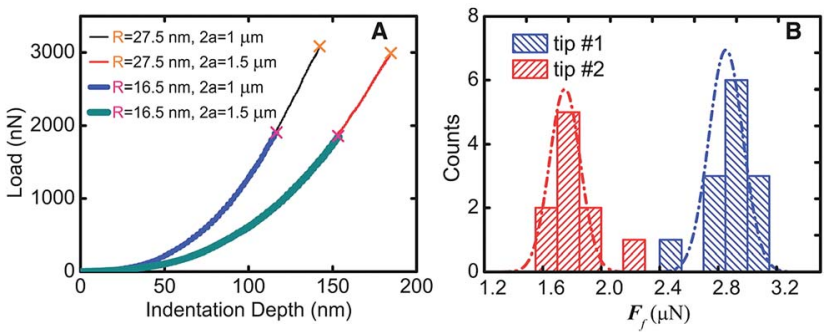

Fig. 6 Fracture test results (A) the test line corresponding to different film diameters and tip radius. (B) Histogram and Gauss distribution of two kinds of fracture forces. ${ }^{9}$

graphene and related materials, ${ }^{\mathbf{1 0 1}}$ it is possible to regulate the size of the energy gap by adjusting the interaction between graphene and substrate materials. ${ }^{\mathbf{1 0 2 - 1 0 5}}$

2.3.4 Magnetic properties of graphene. Graphene does not have $\mathrm{d}$ or $\mathrm{f}$ electrons, but this does not mean that graphene is not magnetic. On the contrary, under certain conditions, graphene can display paramagnetism and even ferromagnetism. In the case of low temperature and a zero magnetic field, the electrical conductivity of graphene exists at a minimum value, and the value is close to $4 e^{2} / h$. Graphene is able to exhibit the quantum Hall effect at room temperature, and it appears anomalous with the quantum Hall effect at low temperatures (below $4 \mathrm{~K}$ ). ${ }^{\mathbf{1 0 6 , 1 0 7}}$ Graphene has characteristics of "pseudo-spin" and "pseudo-magnetic field", which appear as the properties of electron spin and magnetic fields. Electron spin and current injection and the detection of graphene were recently confirmed at room temperature. ${ }^{\mathbf{1 0 8 , 1 0 9}}$

Wang et al. applied various annealing temperatures to oxidize graphene to regain its ferromagnetism at room temperature. ${ }^{110}$ Geim et al. obtained diamagnetic nanographene at room temperature and paramagnetic nanographene based on low temperature (Fig. 10). ${ }^{111}$ Two recent articles have pointed out that doped graphene under various conditions has paramagnetism. ${ }^{112}$

\subsection{Raman spectrum of graphene}

In 2006, Ferrari et al. first proposed the Raman spectroscopy identification method for single-layer graphene (Fig. 11). ${ }^{113}$ The major Raman spectrum characteristic peak for graphene is the $\mathrm{G}$ peak, caused by in-layer transverse vibrations of $\mathrm{sp}^{2}$ hybridized carbon atoms, and this appears in the vicinity of 1580

Table 1 Comparison of the physical properties between several materials and $\mathrm{h}-\mathrm{BN}$

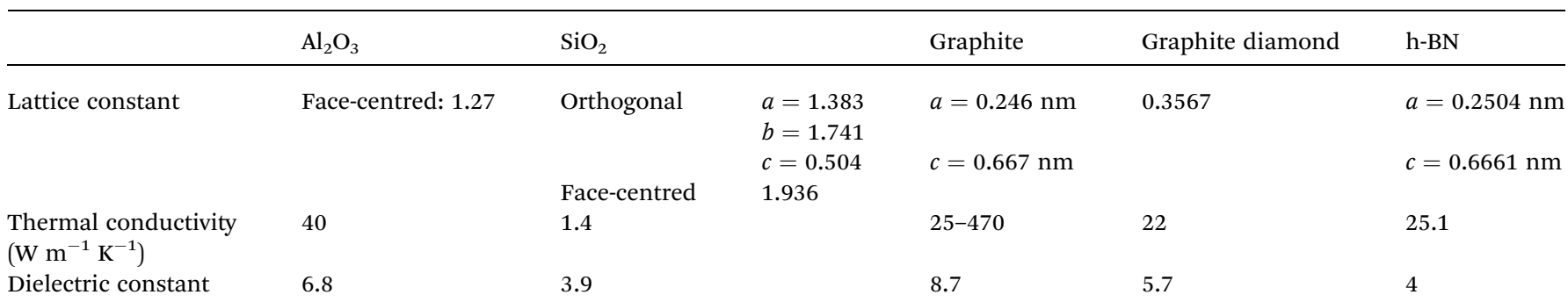



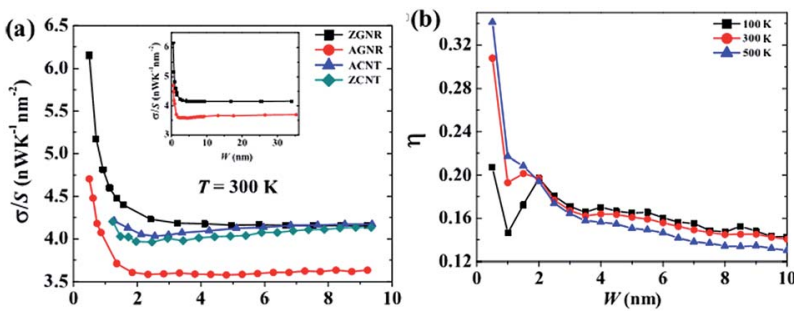

Fig. 7 Different widths of graphene nanosheets' thermal conductivity changes at different temperatures ((a) The thermal conductivity change images of different materials at $300 \mathrm{~K}$. (b) The anisotropy test images of different widths of nanoribbons at different temperatures). ${ }^{91}$

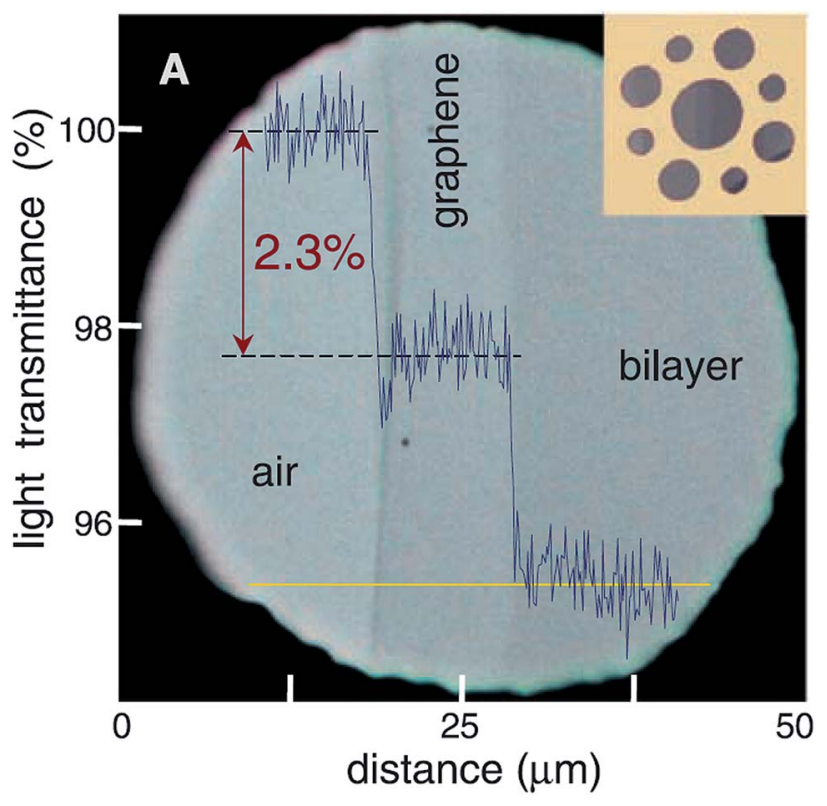

Fig. 8 Looking through one-atom-thick crystals. ${ }^{93}$

$\mathrm{cm}^{-1}$. The peak can effectively reflect the number of layers of graphene sheets, and is vulnerable to stress. With the increase in the number of layers, $N$, the position of the G peak will move to lower frequency, and this displacement of the movement is related to $1 / N .^{114}$ The $\mathrm{G}$ peak is susceptible to doping, and the peak frequency and peak width can be used to detect the doping level. ${ }^{115}$

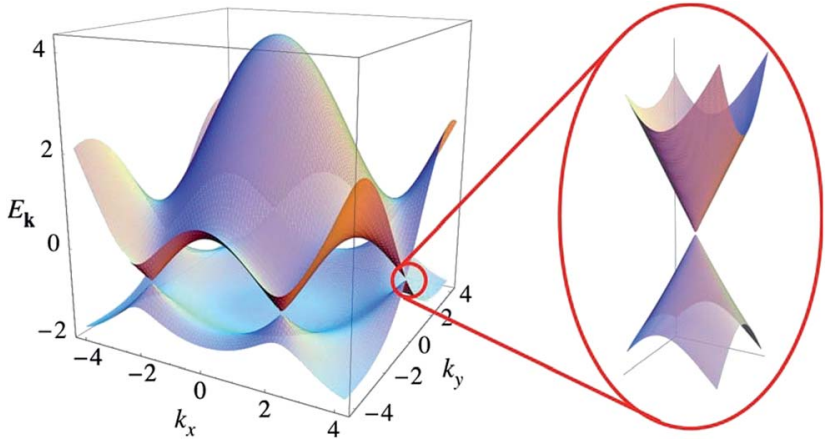

Fig. 9 Electronic dispersion in the honeycomb lattice. ${ }^{96}$
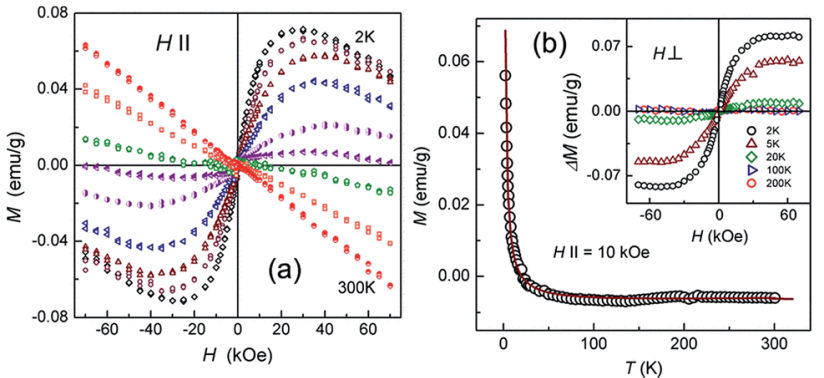

Fig. 10 Magnetic response of graphene ((a) Magnetic moment as a function of magnetic field intensity at different temperatures from 2 $\mathrm{K}$ to $300 \mathrm{~K}$. (b) Magnetic moment images with the changing temperature for the same sample). ${ }^{111}$
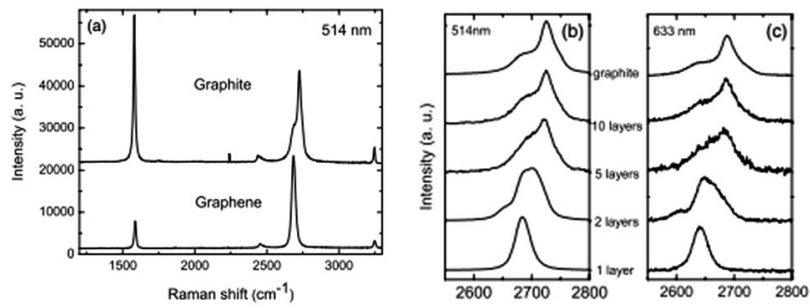

Fig. 11 Raman spectra of ideal graphene and of different layers under different wavelengths. ${ }^{113}$

The D peak usually refers to a disordered vibration peak of graphene. The peak appears at $1270-1450 \mathrm{~cm}^{-1}$, with its specific location related to the excitation wavelength. ${ }^{116}$ The peak is caused by the lattice vibration of graphene keeping away from the centre of the Brillouin zone, and is used to characterize defects or the edge of the graphene sample. The $2 \mathrm{D}$ peak is a two-phonon resonance second-order Raman peak, while the double resonance process connection phonon wave vector and the electronic band makes the frequency of the 2D peak susceptible to excitation wavelength. ${ }^{117}$

Characteristic peaks can be used to determine the different layers of graphene, and, as an optical means of almost zero damage, Raman spectroscopy, which is widely recognized and used by researchers, has greatly enhanced the efficiency of this kind of identification.

\section{Two-dimensional hexagonal boron nitride (h-BN)}

\subsection{Structure of $h-B N$}

As an analogy of graphene, B atoms and $\mathrm{N}$ atoms of a 2D h-BN are alternately arranged to form a honeycomb structure, following the law of a hexagonal lattice formation (Fig. 12). ${ }^{118,119}$ The $\mathrm{B}-\mathrm{N}$ bond length is $1.45 \AA$, which forms through $\mathrm{sp}^{2}$ hybridization. Three $\mathrm{sp}^{2}$ orbits of each $\mathrm{B}$ atom combine with the $\mathrm{sp}^{2}$ orbit of adjacent $\mathrm{N}$ atoms to form a strong $\sigma$ bond, likewise, three $\mathrm{sp}^{2}$ orbits of each $\mathrm{N}$ atom combine with the $\mathrm{sp}^{2}$ orbit of adjacent $\mathrm{B}$ atoms to form a strong $\sigma$ bond. Adjacent layers of $\mathrm{h}-$ $\mathrm{BN}$ are combined with weak van der Waals forces, and in each 


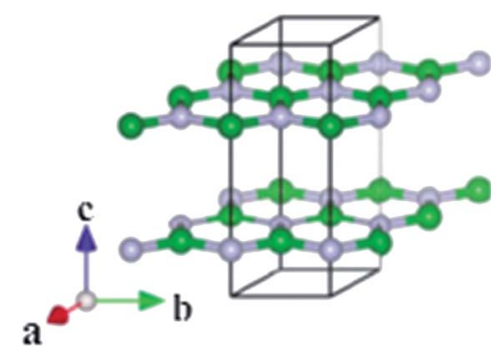

(A)

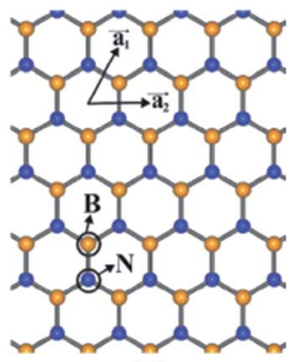

(B)
Fig. 12 Structure of $h-B N$. (A) Stereogram. (B) The 2D floor plan. ${ }^{118}$

layer $\mathrm{B}$ atoms and $\mathrm{N}$ atoms are joined by covalent bonds. The interlayer spacing of graphene is $0.335 \mathrm{~nm}$, and the interlayer spacing of h-BN is $0.333 \mathrm{~nm}$, which is slightly less than that of the graphite. In the $c$-axis direction of h-BN, the bonding force is small and the interlayer spacing is large, making the interlayer slide easily. ${ }^{120-134}$

\subsection{Preparation of $\mathbf{h}-\mathbf{B N}$}

Whether by experimental or theoretical considerations, h-BN crystals have been prepared in many ways. ${ }^{40,133-143}$ Some basic methods include: mechanical separation, ${ }^{38}$ chemical vapour deposition (CVD), ${ }^{133,134}$ aqueous solvent thermal synthesis, ${ }^{135-137}$ solvent stripping and so on (Fig. 13). ${ }^{129,138}$

\subsection{Physical properties of $\mathbf{h}-\mathrm{BN}$}

3.3.1 Electrical characteristics of $\mathbf{h}-\mathbf{B N}$. The surface of a 2D $\mathrm{h}$-BN is smooth, while its lattice structure is very similar to graphene. 2D h-BN has a large optical phonon mode, a wide band gap, no dangling bonds or electron traps on the surface and an local density approximation (LDA) calculated band gap of about $4.5 \mathrm{eV}$ and $\mathrm{GW}$ calculated band gap of $6.0 \mathrm{eV},^{118,139}$ which indicates it belongs to the wide band gap class of
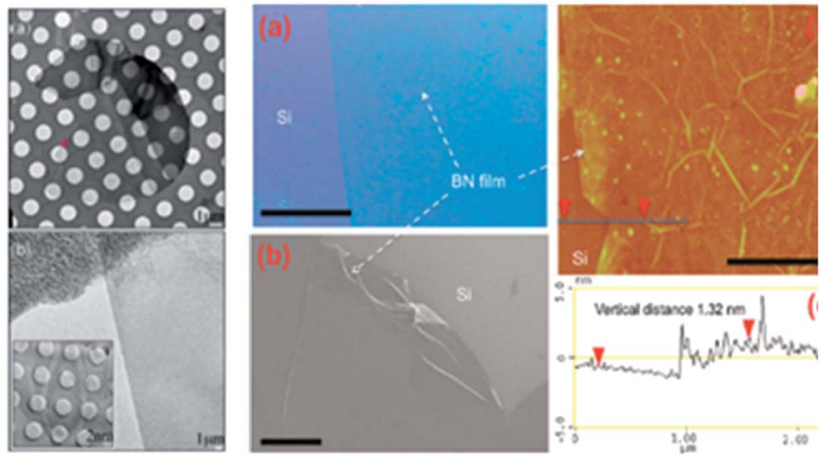

Fig. 13 Preparation methods of $\mathrm{h}-\mathrm{BN}$ with the mechanical stripping method and the CVD method (The left group figures: (a) low magnification and large defocus of the h-BN TEM images. (b) The folding images for $\mathrm{h}-\mathrm{BN}$. The right group figures: (a) Photograph of a large $\mathrm{h}$-BN film on a silicon substrate. Scale bar is $1 \mathrm{~cm}$. (b), SEM image shows a h-BN film (scale bar $10 \mu \mathrm{m}$ ). (c and d) AFM image and line-scan profile indicate that $\mathrm{h}$-BN film has uniform thickness of a 1 $\mathrm{nm}$. Scale bar is $2 \mu \mathrm{m}){ }^{38,133}$
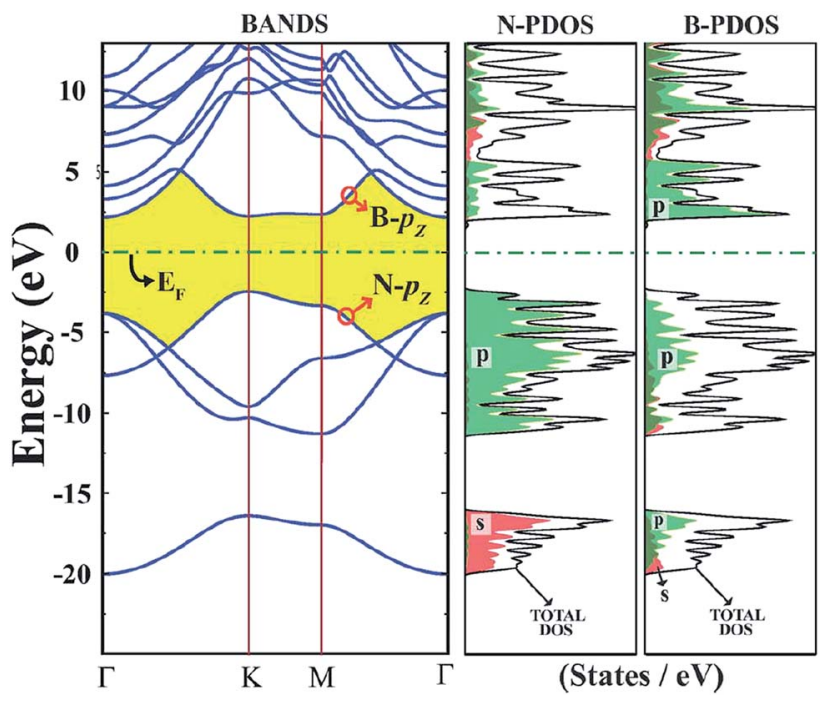

Fig. 14 Orbital property and electronic structure of h-BN. ${ }^{118}$

insulators. Fig. 14 is a band structure of $2 \mathrm{D}$ h-BN. ${ }^{118}$ As Fig. 14 shows, h-BN has a direct band gap, and both the top and rewinding bottom of the valence band are in the high symmetry $K$ point. The HOMO and LUMO levels of the system are determined by $\pi$ and $\pi^{*}$ located on the $\mathrm{N}$ atom and B nucleus, respectively. ${ }^{118}$

3.3.2 Force thermal characteristics of $\mathbf{h}-\mathbf{B N}$. The mechanical properties of $2 \mathrm{D} \mathrm{h}-\mathrm{BN}$ are prominent. ${ }^{140,141}$ Song et al. measured the mechanical properties of h-BN film by using a diamond tip to emboss the h-BN film centra. ${ }^{133}$ As shown in Fig. 15 , E2D is a post-elastic coefficient, and $2 \mathrm{D} \mathrm{m} \sigma$ is the pretensioned stress. A large number of experimental results and theoretical calculations have shown that the Young's modulus of the 2D h-BN is large (approximately $270 \mathrm{~N} \mathrm{~m}^{-1}$ ).

At room temperature, the thermal conductivity of h-BN is up to $400 \mathrm{~W} \mathrm{~m}^{-1} \mathrm{~K}^{-1}$, which is higher than the majority of metals and ceramic materials. h-BN has a typical anisotropy: having a high thermal conductivity in the direction perpendicular to the $c$-axis of $300 \mathrm{~W}\left(\mathrm{~m}^{-1} \mathrm{~K}^{-1}\right)$, low thermal expansion coefficient of $0-2.6 \times 10^{-4} \mathrm{~K}^{-1}$, and a relatively high tensile strength (41 MPa). When parallel to the $c$-axis direction, h-BN has a lower thermal conductivity of $20-30 \mathrm{~W}\left(\mathrm{~m}^{-1} \mathrm{~K}^{-1}\right)$ and high compressive strength, and so on. ${ }^{138,142-145}$

3.3.3 Optical properties of $\mathbf{h}-\mathrm{BN}$. In both the experimental and theoretical calculations, $2 \mathrm{D}$ h-BN has no absorption in the visible range, but has absorption spectroscopy in the ultraviolet region and a good photoluminescence property. Geim et al. changed the silicon-rays to detect the polishing process, using an optical microscope to view a single atomic layer thickness of h-BN. ${ }^{1}$ The electronic state of the materials can be reflected by the optical absorption properties, ${ }^{35}$ which are widely used to calculate the band gap of semiconductors. We know the range from 3.6 to $7.1 \mathrm{eV}$ of the h-BN band gap energy from the experiments of h-BN with different structures reported in the available literature data. ${ }^{131}$ 


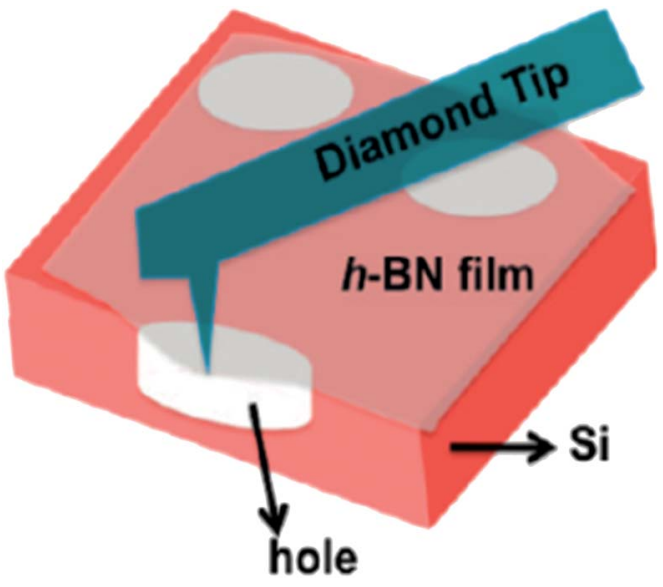

Fig. 15 Schematic of nanoindentation on a suspended h-BN membrane. ${ }^{133}$

Gao et al. measured the UV-vis optical absorption spectrum of h-BN by investigating as-prepared h-BN nanosheets. From Fig. 16, we can see that the absorption peaks around 251, 307 and $365 \mathrm{~nm}$ corresponds to a band gap energy of 4.94, 4.04, and $3.40 \mathrm{eV}$, respectively. These two absorption peaks at 307 and $365 \mathrm{~nm}$ are due to the optical transitions and the redistribution of h-BN's electron-hole density between van Hove singularities of excited state. ${ }^{\mathbf{1 3 1}}$ As a wide band gap material, ${ }^{35,146} \mathrm{~h}-\mathrm{BN}$ is transparent in infrared and visible light, while in ultraviolet light it has a strong optical absorption at $251 \mathrm{~nm}$ with a strong exciton peak. ${ }^{\mathbf{1 3 1}}$

3.3.4 Magnetic properties of h-BN. $2 \mathrm{D} h-\mathrm{BN}$ is a nonmagnetic semiconductor with a wide band gap. ${ }^{146}$ Studies have found that the introduction of doping or defects to $2 \mathrm{D} h$ $\mathrm{BN}$ systems can lead to spontaneous magnetization of h-BN. Wu et al. calculated the condition that $\mathrm{B}$ atoms and $\mathrm{N}$ atoms are replaced by carbon atoms, ${ }^{\mathbf{1 4 7 , 1 4 8}}$ resulting in the generation of spontaneous magnetism in 2D h-BN (Fig. 17). Si et al. found that the vacancy defects of $\mathrm{B}$ atoms or $\mathrm{N}$ atoms in h-BN can also

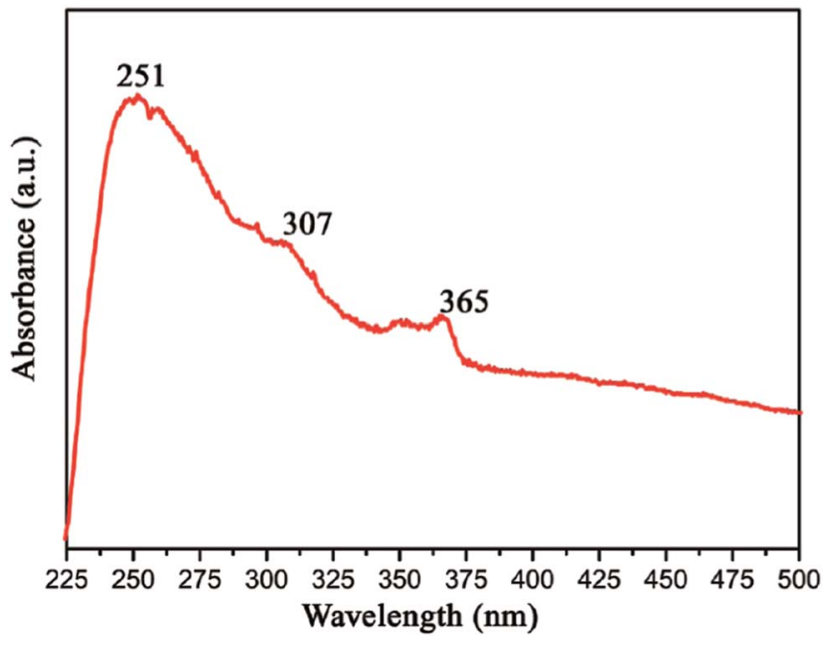

Fig. 16 UV-vis optical absorption spectra of BN. ${ }^{131}$
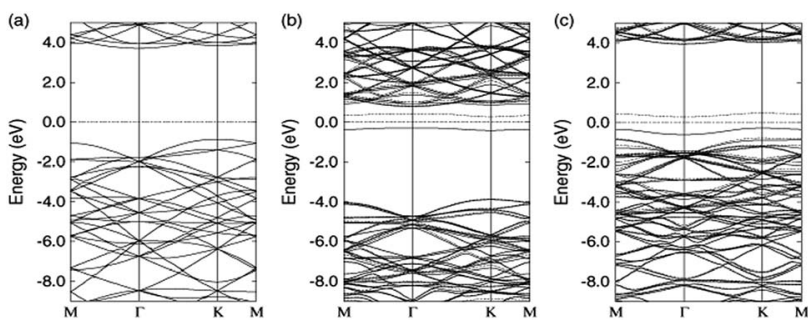

Fig. 17 Calculated band structures of the pristine graphitic-BN with different atoms. (a) with a boron atom (b) and a nitrogen atom (c) substituted by a carbon atom. ${ }^{148}$

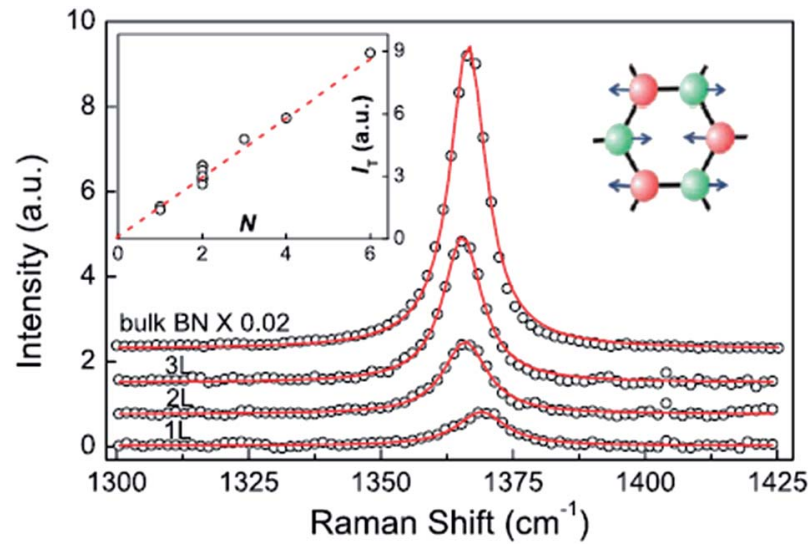

Fig. 18 Raman spectra of 2D h-BN. ${ }^{152}$

lead to spontaneous spin magnetization. ${ }^{\mathbf{1 4 9}}$ These studies suggest that boron nitride nano-ribbons can present excellent half-metallic magnetism in a variety of states. ${ }^{\mathbf{1 5 0}}$

By researching no passivation and passivation with zigzag boron nitride nano-ribbons, Lai et al. found that its system presents as semi-metallic, ${ }^{150}$ while the nitrogen atom edge passivation is not magnetic. V. Barone et al. found no spontaneous spin polarization in passivated serrated boron nitride nano-ribbons, ${ }^{\mathbf{1 4 6}}$ while under the effect of an external electric field, the material can realize a metal-semiconductor-semimetal transition. F. W. Zheng et al. found that when the B atoms are edge passivated, ${ }^{151}$ the electronic polarization rate at the Fermi level is $100 \%$, showing semi-metallic properties with a band gap of $0.38 \mathrm{eV}$, while the conductivity of the system is decided by metallic spin electrons. Also, when the $\mathrm{N}$ atoms are edge passivated, the antiferromagnetic structure is more stable than the ferromagnetic structure, as the energy of the former is approximately $33 \mathrm{meV}$ per (edge atom) more than that of the latter.

\subsection{Raman spectroscopy of $h-B N$}

The Raman characteristic peaks of h-BN crystals is at about $1366 \mathrm{~cm}^{-1}$, and a single-layer peak will blue-shift $4 \mathrm{~cm}^{-1}$ due to the $\mathrm{BN}$ bond of a single-layer h-BN having slightly shorter phonon modes, causing $\mathrm{E}_{2 \mathrm{~g}}$ to harden (Fig. 18). ${ }^{152}$ The red-shift depends on random strain introduced by the stripping process, which dominates in the double h-BN. Therefore, the h-BN Raman characteristic peak is red-shift $1-2 \mathrm{~cm}^{-1}$. 


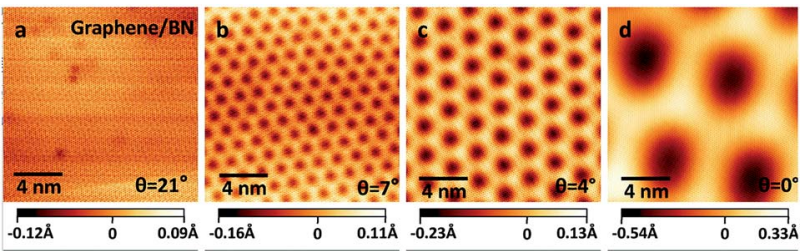

Fig. 19 STM pictures of graphene/h-BN heterostructure with different rotational orientations. ${ }^{70}$

\section{Composite structure of graphene/ $\mathrm{h}-\mathrm{BN}$}

\subsection{Research into graphene/h-BN heterojunction structures}

The linear dispersion relationship of graphene near the Dirac point is responsible for its high carrier mobility characteristic, which has attracted wide attention (Fig. 19) ${ }^{70}$ and is considered an important characteristic for electronic devices utilizing carbon Keener materials. ${ }^{\mathbf{1 0 6 , 1 5 3 , 1 5 4}}$ However, its zero band gap has hindered graphene's application in the construction of nanoelectronic devices, because these devices require a valid strip gap. Thus, whether graphene can open an effective band gap becomes critical. Researchers have used a variety of methods to achieve this objective. For example, hydrogenated graphene, ${ }^{\mathbf{1 5 5}}$ isoelectronic co-doping of $\mathrm{B}$ and $\mathrm{N}$ atoms, ${ }^{156}$ adsorption of metal atoms, ${ }^{157}$ double extra electric field components, ${ }^{2,158}$ a graphene-substrate interaction, ${ }^{159}$ the level of the tensile stress and so on. ${ }^{71}$ All the methods were double nanostructures, which makes them exceptionally compelling, because the interaction between the layers and stacking mode provides an additional degree of freedom to regulate the electronic structure. $\mathrm{C}$ materials and h-BN have matched lattice constants. By forming a graphene/h-BN heterostructure to regulate graphene's electronic structure and energy band, the goal of applying graphene to optoelectronic devices is achieved.

In 2000, Oshima et al. ${ }^{\mathbf{1 6 0}}$ prepared two-layer structured graphene/h-BN in the Ni(111) direction, and studied the influence of monolayer graphene on the Ni(111) substrate grown h$\mathrm{BN}$ single-layer electronic structure, ignoring the effects of h-BN on the electronic structure of graphene. Stewart et al. calculated the most stable configuration of graphene/h-BN electronic properties through the tight-binding and density functional theory. ${ }^{161}$ Their research showed that a level perpendicular to the applied electric field could regulate the electronic structure of the whole system, including the offset, anti-cross, and other forms of energy deformation, and could be used to control the size of the band gap according to different needs. In 2010, Bjelkevig et al. used the CVD method on Ru's (0001) direction to prepare a double heterojunction structure graphene/h-BN. ${ }^{\mathbf{1 6 2}}$ The study found that there was a strong charge transfer between the $\mathrm{BN}$ and graphene, which occupied part of the $\pi^{*}$ band, and made the occupied $\sigma^{*}$ closer to the Fermi level. This showed that forming a double heterojunction structure of graphene/h$\mathrm{BN}$ could regulate the electronic structure of graphene. In 2012, Tang et al. made single-crystal graphene grow on h-BN, ${ }^{163}$ although the growth size was not large, and then used Raman technology to demonstrate that the nucleation growth of hexagonal graphene involved a single crystal structure. This finding enriched the means of preparing graphene boron nitride heterojunctions, and makes the rapid preparation of high-quality graphene/h-BN possible. Especially recently, Tang et al. used the CVD method to make graphene from graphene/h$\mathrm{BN}$ in an efficient large area, ${ }^{\mathbf{1 6 4}}$ such that the preparation of graphene reached a new level. Meanwhile, the applications of graphene/h-BN heterostructures in nanodevices has gained technical support.

\subsection{Composite mode structure of graphene/h-BN}

4.2.1 Stacking of graphene/h-BN heterostructures. A graphene/h-BN heterostructure is formed by the $1 \times 1$ lattice matched stacking of monolayer graphene and h-BN. The major three stacking patterns in most studies are: (1) AA stacking, which means $\mathrm{C}$ atoms are positioned immediately above the $\mathrm{B}$ and $\mathrm{N}$ atoms; (2) $\mathrm{AB}$ stacking, which means one $\mathrm{C}$ atom is positioned above the $\mathrm{N}$ atom, while the other $\mathrm{C}$ atom is positioned above the h-BN ring; (3) AB stacking, which means one $\mathrm{C}$ atom is positioned above the $\mathrm{B}$ atom, while the other $\mathrm{C}$ atom is positioned above the h-BN ring.

Zhong et al. verified that the $\mathrm{AB}$ stacking of the graphene/h$\mathrm{BN}$ double-layer heterogeneous nanostructure was the most stable by first-principles calculations, and found that as stress increases, the band gap increases linearly. ${ }^{165}$ Fan et al. applied LDA and PBE + VDW methods to study the structural energy variations of the graphene/h-BN double-layer heterogeneous
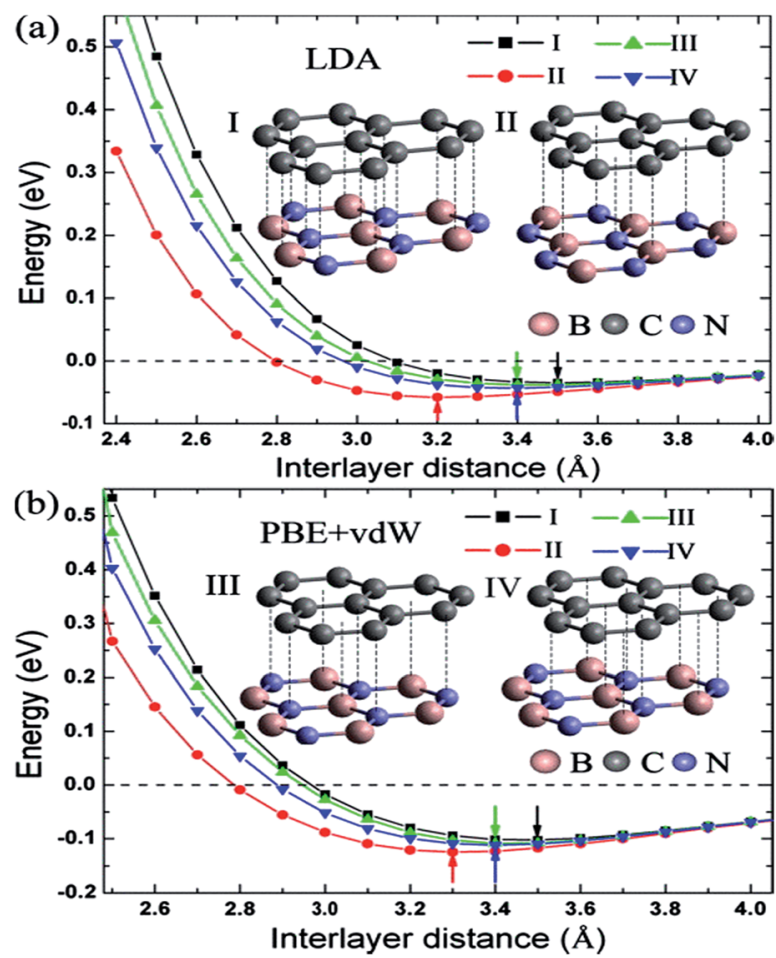

Fig. 20 BE per unit cell of graphene/h-BN with different stacking manners. ${ }^{166}$ 
nanostructures of four stacking patterns with various interlayer distances. ${ }^{166}$ The results showed that with either method, AB stacking, where $\mathrm{N}$ atoms are positioned towards the centre of the graphene hexagon and $\mathrm{B}$ atoms are positioned towards the $\mathrm{C}$ atoms, is most stable at various interlayer distances, with the stable model and calculation results shown in Fig. 20. They studied four kinds of stacking configurations: I-IV. I had a hexagonal configuration (AA), where $\mathrm{C}$ atoms are positioned above the $\mathrm{B}$ atoms or $\mathrm{N}$ atoms. II and III had a Bernal configuration $(\mathrm{AB})$, where $\mathrm{C}$ atoms of one sub-lattice of graphene are positioned above the $\mathrm{N}$ atoms (configuration II) or $\mathrm{B}$ atoms (configuration III), while $\mathrm{C}$ atoms of the other sub-lattice of graphene are positioned above the centre of the $\mathrm{BN}$ hexagon. Configuration IV can be obtained from the translation of the h$\mathrm{BN}$ layer in configuration I along the direction of the $\mathrm{C}-\mathrm{C}$ bonds by the distance of $1 / 6$ graphene lattice constant. ${ }^{166}$ Kan et al. also found that the band gap of the graphene/h-BN double-layer heterogeneous $\mathrm{AB}$ stacking stable structure could be modulated through the interlayer distances by first principles. ${ }^{\mathbf{1 6 7}}$

Utilizing the VASP package local density approximation (LDA), the changes of binding energy (BE) for each original cell with the variations of the interlayer distance were calculated, ${ }^{\mathbf{1 6 8}}$ and the results showed that various stacking structures had different equilibrium positions, specifically, different structure balancing interlayer spacing of 3.5, 3.2, 3.4 and $3.4 \AA$ for configurations I-IV, respectively, which were consistent with previous calculations for the deposition of graphene on boron nitride. ${ }^{71}$ For this double-layer heterostructure, the order of stability under the same interlayer spacing was: configuration I $<$ configuration III < configuration IV < configuration II. This phenomenon is related to the attractive interaction between the electrons in graphene and the cations in $\mathrm{BN}$ and to the repulsive interaction between the electrons in graphene and the anions in $\mathrm{BN}$; while $\mathrm{N}$ anions tend to be directly above the centre of the $\mathrm{C}$ hexagon, because the electron density of this position is low,
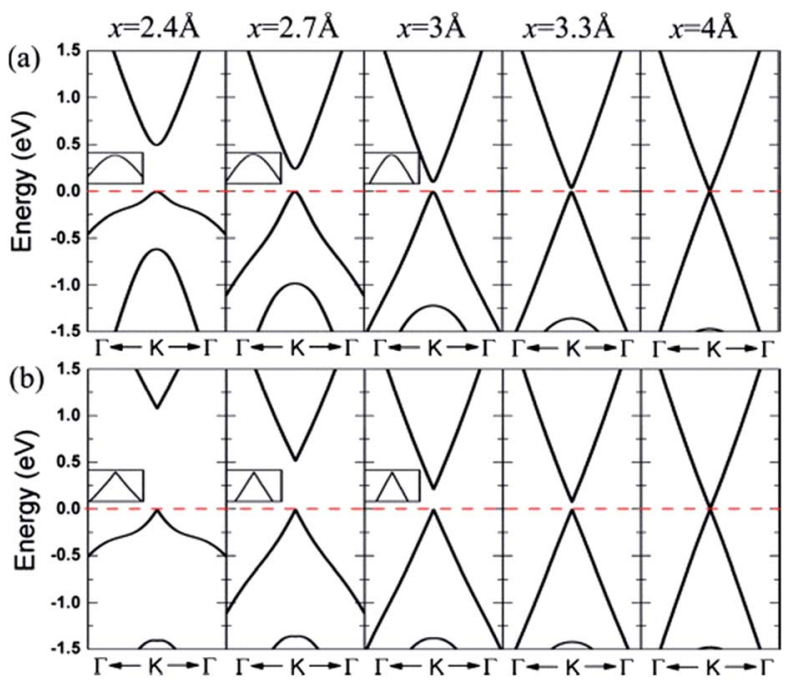

Fig. 21 Band lines near the Fermi level of C/BN HBLs with interlayer distances $x=5,3.3,3,2.7$, and $2.4 \AA$ for (a) configuration II and (b) configuration IV. The Fermi level is set to zero. ${ }^{166}$ and $\mathrm{B}$ cations tend to be directly above $\mathrm{C}$ atoms to enhance the effect of attracting (configuration II).

4.2.2 Relations between graphene/h-BN heterostructure's configuration, spacing and electronic structure properties. The results show that configurations I-III have similar energy band structures. Fig. 21(a) presents, under various interlayer distances, the energy band structure of configuration II near the Dirac point. The energy band structure of configuration IV is shown in Fig. 21(b). ${ }^{166}$

When the interlayer distance is large enough, the energy band structure near the Fermi level is consistent with that of monolayer graphene, because the interlayer interaction under such a distance is very weak. With the decrease of the distance between the layers, the interlayer interaction increases, resulting in a reconstruction of HBL's charge. Due to the different chemical environments, the equivalence between the two different sub-lattice of graphene is destroyed, and a small band gap is opened up at the Dirac point between the valence and conduction bands. Obviously, the smaller the interlayer distance, the bigger the opened band gap, which thus provides a possible approach for the band gap regulation of graphene.

For HBLs of configuration I-III, at the Dirac point, there is a parabolic dispersion relationship (nearly free electrons) between the valence and conduction band near the Fermi level, which means the effective mass of electrons is non-zero. However, for HBLs of configuration IV, a band gap is opened near the Dirac point, and the energy band structure maintains the linear dispersion relationship with graphene, which means the effective mass of electrons and holes is zero. The high mobility of carriers in this heterogeneous double layer, of which the band gap is not zero, is comparable to that in the graphene monolayer, which is significant for the preparation of graphene devices that require both a band gap and high carrier mobility.

The variation of the band gap of the HBLs can be studied by adjusting the interlayer distance, as shown in Fig. 22. ${ }^{167}$ The band gap increases progressively with the decrease of the interlayer distance, but the extent of change is different for the

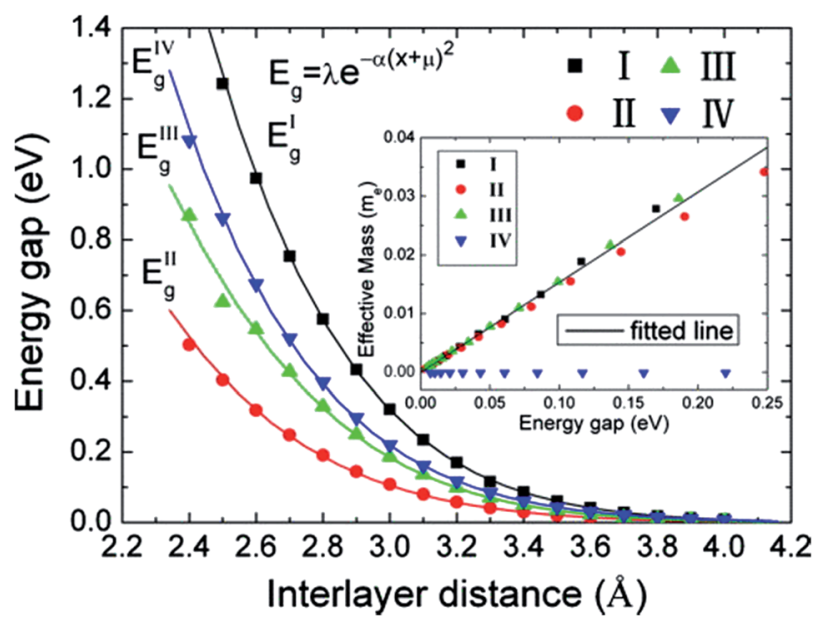

Fig. 22 Variation in the energy gap of HBLs as a function of interlayer spacing. The inset shows the variation of the effective masses $v s$. the band gap. ${ }^{166}$ 

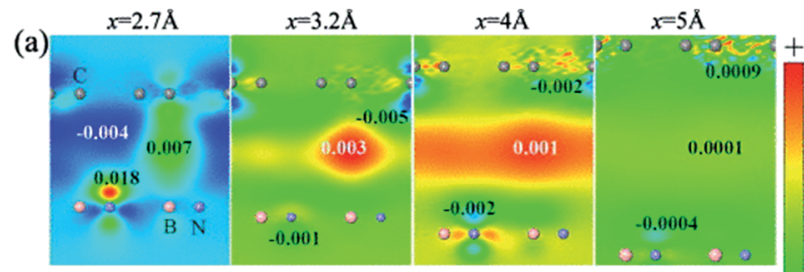

(b)

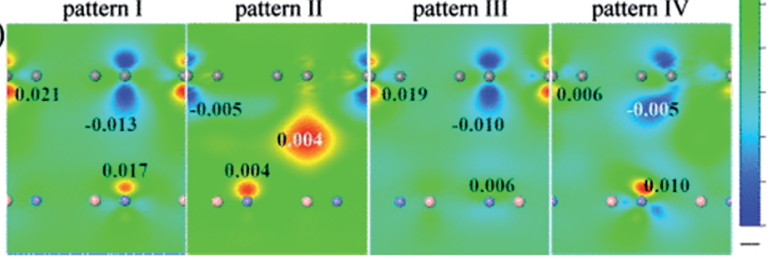

Fig. 23 Contour plots of the charge density difference (a) for stacking pattern I at the interlayer distances $x=2.7,3.2,4$ and $5 \AA$, respectively, and $(b)$ for the four stacking patterns $(I-I V)$ at the interlayer distance $x$ $=3 \AA .{ }^{198}$

four configurations. Under the same interlayer distance, configuration I and II have the maximum and minimum band gap, respectively, and the other two configurations have the medium band gap.

From Fig. 23, we can see that the charge density near the two sub-lattice of graphene is no longer equivalent, and as the interlayer distance decreases, this asymmetry becomes more pronounced. This shows that when the interlayer distance is compressed, the difference between the potential energy of the two sub-lattices of graphene becomes larger. Furthermore, for the four configurations, the degree of sensitivity to the change of the interlayer distance is related to the atomic arrangement of the HBLs. The carbon atom of configuration I is exactly located above the $\mathrm{B}$ atom or $\mathrm{N}$ atom of the $\mathrm{BN}$ monolayer, causing the highest asymmetry of the two graphene sub-lattices, and therefore configuration $\mathrm{I}$ is the most sensitive to the interlayer distance. The $\mathrm{C}$ atoms of one sub-lattice of configuration I are located directly above the B atoms; however, all the $\mathrm{C}$ atoms are symmetrically distributed around the $\mathrm{N}$ atoms. In this arrangement, the asymmetry of the two carbon atoms sublattice is the lowest, so configuration II is the least sensitive to the interlayer distance. This is also consistent with the trends in charge density with the variation of interlayer distance for the four configurations, as shown in Fig. 23(b).

DFT calculations show that for the graphene/h-BN HBLs, the band gap and effective mass of carriers can be regulated by changing the interlayer distance and stacking patterns. With the decrease of the interlayer distance, the band gap gradually increases. With the increase of the band gap, for $\mathrm{AA}$ and $\mathrm{AB}$ stacking patterns (configurations I-III), the effective mass of carriers linearly increases; for configuration IV, the linear dispersion relation in the monolayer graphene reserves in graphene/h-BN HBLs. The quality of controllable band gap and the small effective mass (even zero for configuration IV) of C/ BN HBLs support it having potential important applications in the construction of high-performance graphene nanodevices.
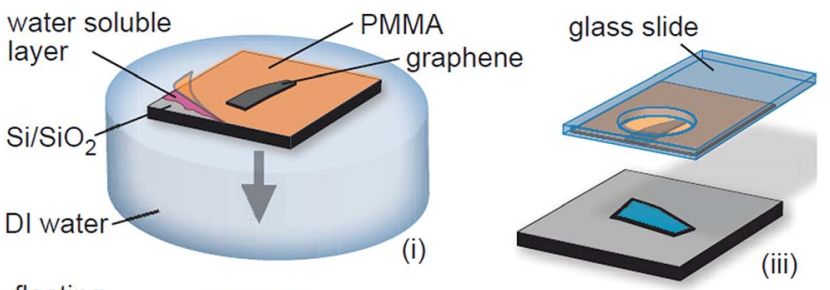

floating
PMMA
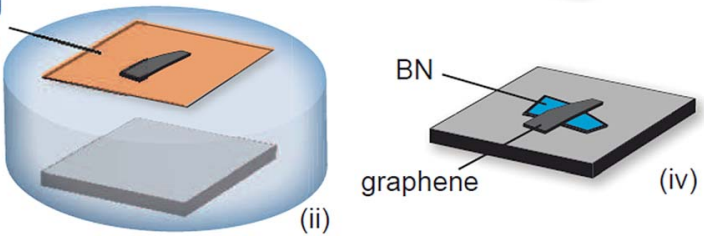

Fig. 24 Schematic of the transfer process to fabricate graphene-onBN devices. ${ }^{72}$

\subsection{Preparations of graphene $/ \mathrm{h}-\mathrm{BN}$ heterostructures}

From 2010 to today, the methods of preparing graphene/h-BN heterostructures have become a hot topic of research and more and more reliable methods have been developed.

Wet transfer method. Dean et al. first prepared a graphene transistor device with high mobility by transferring mechanically stripped graphene to the h-BN substrate and then using the h-BN as the support substrate for the graphene (Fig. 24). ${ }^{72}$ Its electron mobility was measured to be one order of magnitude higher than silica in the same conditions, and a clear graphene quantum Hall effect was observed. This method involved graphene in contact with an aqueous solution. Therefore it is referred to as "wet transfer".

Liquid phase exfoliating method. Gao et al. ${ }^{169}$ used liquid phase exfoliating to achieve van der Waals structures of different layers of h-BN and graphene, and observed that the energy level of the hybrid structure was affected by the number of layers and the stacking mode of the two layers (Fig. 25).

These two methods inevitably have impurities appear on the top and bottom of the graphene, a low success rate of the lattice

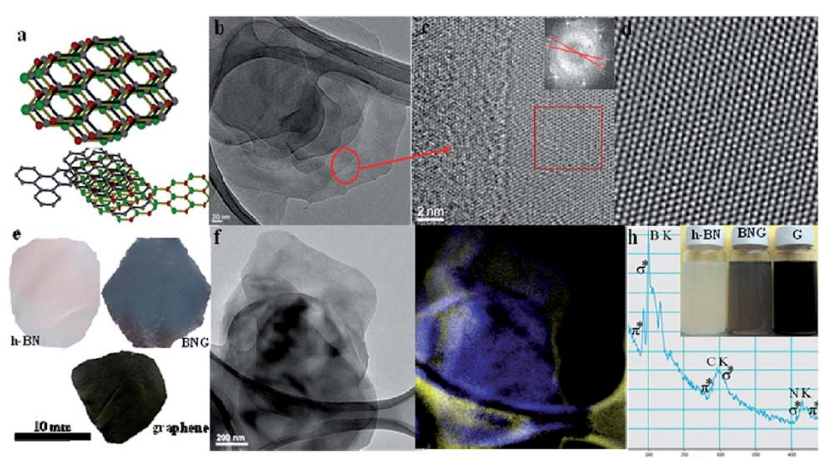

Fig. 25 Schematic of the liquid phase exfoliating method.(a) The illustration of the stacked structure of graphene and h-BN nanosheets. (b) -(d) The TEM images of stack structure with different resolutions. (e) Graphene, h-BN and BNG hybrid free standing films. (f) The h-BN/ GTEM image for mapping. (g) Boron mapping of image (f). (h) EELS spectrum of h-BN/graphene hybrid, $\mathrm{K}$-shell excitations of $\mathrm{B}, \mathrm{C}$, and N. ${ }^{169}$ 
(a)

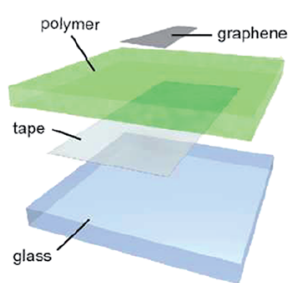

(c)

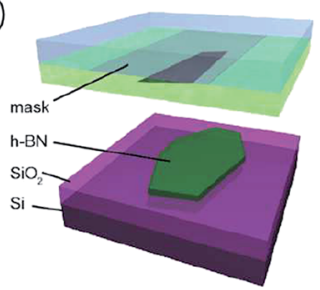

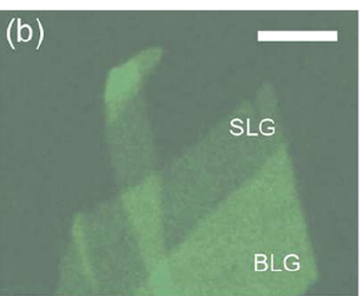

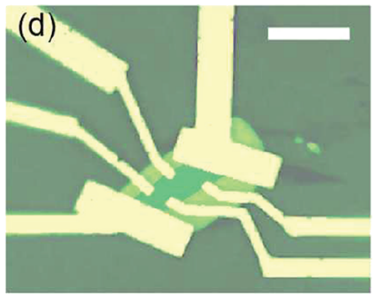

Fig. 26 Dry preparation of graphene/h-BN as a schematic and sample image: (a) schematic of the transfer mask. (b) SLG and BLG areas on the mask, the scale bar equals $5 \mu \mathrm{m}$. (c) Graphene alignment and transfer to a h-BN crystal. (d) Graphene flake in a Hall bar geometry on h-BN. ${ }^{170}$

corresponding paste, poor precision, and the product is easy to fold. In the preparation of this method, the larger the boron nitride, the higher the preparation success rate; the larger the graphene, the easier the device preparation, which is tough in the mechanical stripping process.

Dry transfer method. In order to eliminate the graphene problems encountered in the transfer of folds, in 2011, Zomer et al. ${ }^{170}$ designed a method of the preparation of graphene/h-BN heterostructures (Fig. 26). Similarly, Leon et al. used this method to prepare heterostructures in 2014, and performed the corresponding tests. ${ }^{171}$ From the method and the test results, the only drawback of this method is that it is very difficult to

(a)

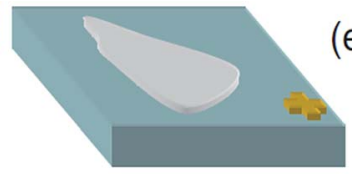

(b)

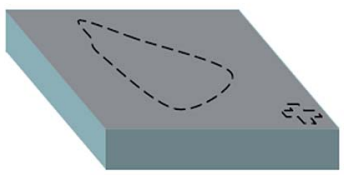

(c)

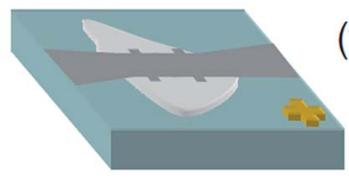

(d)

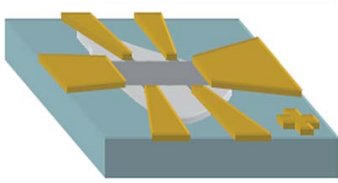

(f)

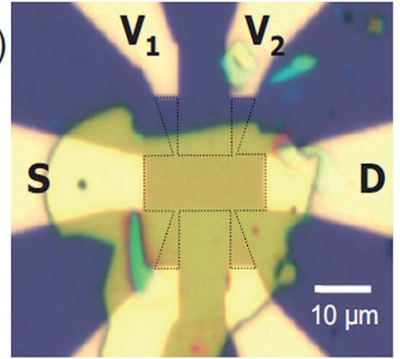

Fig. 27 (a)-(d) Sample fabrication steps shown schematically. (e) SEM image of graphene on a candidate h-BN flake. (f) Optical image of a finished graphene-on-BN device, with the electrodes labelled and the graphene outlined. ${ }^{176}$

find the graphene without the silicon oxide substrate, so that the rate of graphene preparation is very low, and the quality and efficiency are difficult to coexist.

Chemical vapour deposition method (CVD). The researchers used CVD with the adjustment of the order of preparation to generate an artificial hybrid structure of graphite and $\mathrm{h}$ BN. ${ }^{172-175}$

W. Gannett et al. prepared a graphene/h-BN heterojunction by using CVD and run appropriate tests in 2011. After that, many researchers gradually improved the preparation method and made the heterostructure grow faster and faster, with the quality also getting better and better (Fig. 27). ${ }^{176}$

Liu et al. realized the superposed growth of graphene and $\mathrm{h}$ BN by a two-step gas-phase process. ${ }^{177}$ Subsequently, Yan et al. used PMMA as a solid carbon source to achieve the growth of bilayer graphene on a chemically vapour-grown h-BN substrate. ${ }^{178}$ Tang et al. ${ }^{179,180}$ used methane as gaseous carbon source on a h-BN substrate to achieve graphene lamellar nucleation growth with a superlattice structure. ${ }^{164}$ Recently, Kim et al. synthesized graphene/h-BN heterostructures with different hybridization modes by modifying the vapour-phase growth process. ${ }^{174}$ In 2016, Song et al. used PMMA particles to control the nucleation density and grain size, which provides the technical direction for the high-quality mass production of graphene/h-BN. ${ }^{181}$

Transition metal catalysis. In 2010, Cameron Bjelkeving first deposited an atomic layer h-BN on the Ru (0001) surface by an atomic deposition method (Fig. 28), ${ }^{162}$ and then used CVD to grow a layer of graphene on the h-BN surface to form GNR/h-BN/ $\mathrm{Ru}$ (0001) heterostructures, but whether the graphene was directly grown on the h-BN or was due to the role of the Ru underneath the h-BN was not clear and the growth mechanism was not figured out. Oshima et al. achieved the growth of graphene/h-BN/Ni heterostructures in a $\mathrm{Ni}$ (111)-transition metal-catalyzed process in 2000. ${ }^{160}$
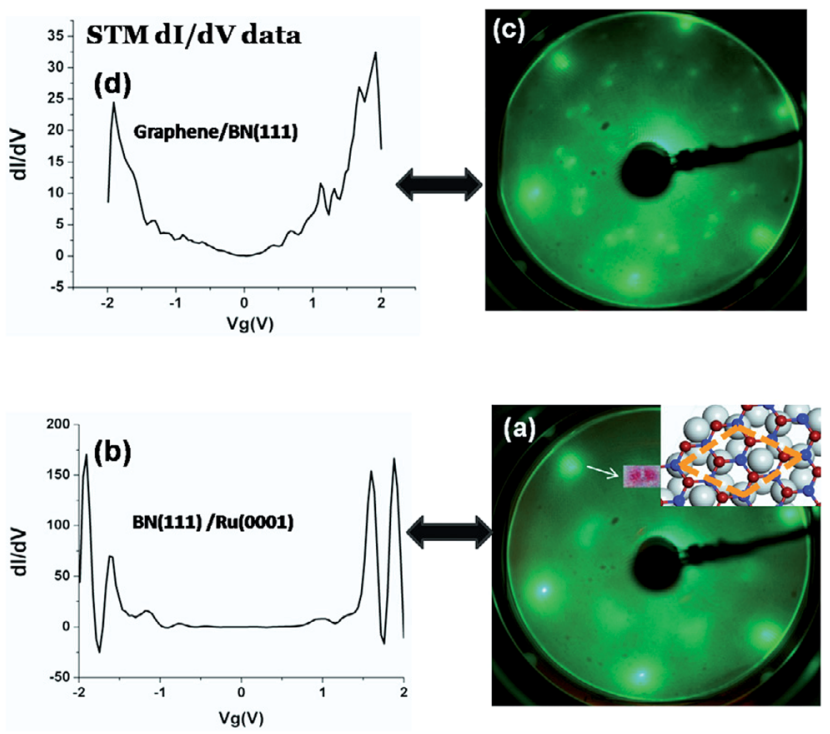

Fig. 28 LEED and STM d//dV data for BN monolayer and graphene/h$\mathrm{BN}$ heterojunction grown on $\mathrm{Ru}(0001) .{ }^{162}$ 

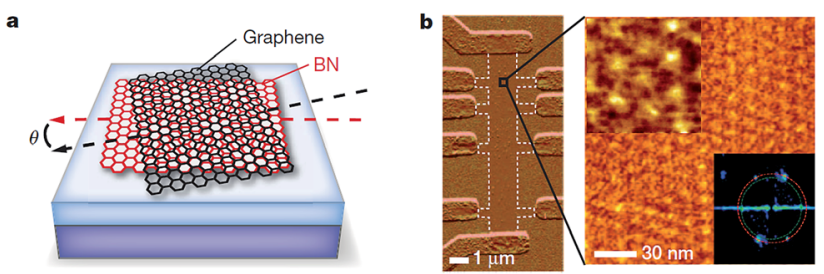

Fig. 29 Moire' superlattice of graphene/h-BN. (a) The schematic diagram of moiré pattern for graphene on $h-B N$. (b) AFM image of a multiterminal Hall bar and a high-resolution image of a magnified region. ${ }^{185}$

Physical transfer method. In 2011, Ponomarenko et al. combined graphene crystals and h-BN thin layers by a physical transfer technique to fabricate heterojunction devices with two monolayer graphene crystals. ${ }^{73}$ Afterwards, through the improvement of the physical transfer technique, researchers successfully fabricated more complex heterostructures and superlattice structures of graphene/h-BN. ${ }^{75,182,183}$ In particular, two research groups successfully observed the novel Hofstadter Butterfly phenomenon in graphene/h-BN heterostructures (Fig. 29). ${ }^{\mathbf{1 8 4 - 1 8 6}}$

The physical transfer method can realize the transfer of graphene on the surface of h-BN. However, this technique also brings issues of structural inhomogeneity and interfacial contamination. Now, researchers are exploring further optimized methods.

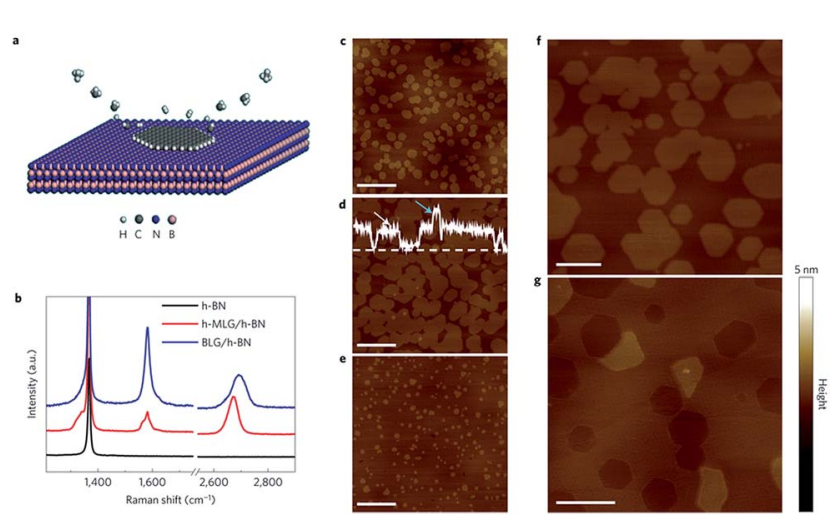

Fig. 30 The method of epitaxial graphene growth and the AFM image. $^{187}$

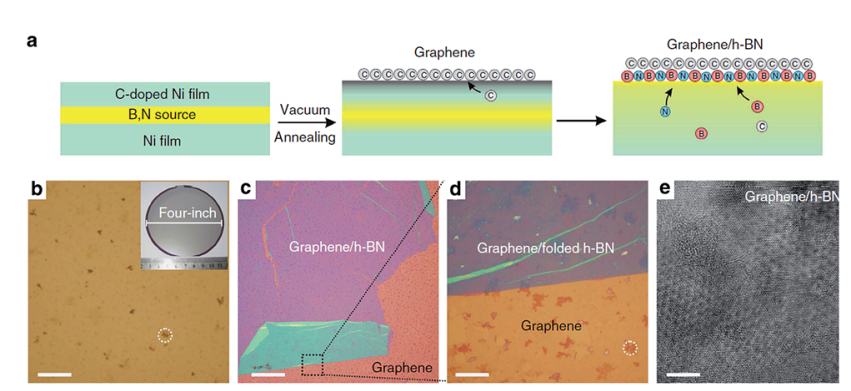

Fig. 31 Growth of graphene/h-BN-stacked heterostructures: schematic illustration and optical images of the as-grown sample on the growth substrates. ${ }^{188}$
Gas-phase epitaxy technology method. In 2013, Yang et al. implemented a controlled van der Waals heterostructures epitaxial growth of graphene on an h-BN inactive substrate by using methane as a gas source and a remote plasma enhanced vapour-phase epitaxy technique (Fig. 30). ${ }^{187}$

Co-segregation method. In 2015, Zhang et al. introduced a method, named 'Co-Segregation Method', which involved large growth directly on the h-BN to produce the desired heterostructures (Fig. 31). ${ }^{\mathbf{1 8 8}}$

\subsection{Properties of graphene/h-BN heterostructures}

Graphene/h-BN heterostructures have attracted much attention because of their novel electrical, morphological, optoelectronics, mechanical and thermal properties.

4.4.1 Optical characteristics of graphene/h-BN heterostructures. A 2D crystal has a large specific surface area, while 2D graphene and h-BN have honeycomb structures. However, the optical and electrical properties of the two materials are very special but completely different. Therefore, when graphene/h$\mathrm{BN}$ heterostructures appeared, researchers quickly focused on the electro-optical properties of the structure. h-BN is a typical material, which is only excited luminescently in the ultraviolet region, ${ }^{35,131,189,190}$ while graphene is excited in the visible region. ${ }^{\mathbf{9 2 , 9 3}}$ Therefore, the components of a graphene/h-BN heterostructure have little influence on each other from an optical properties standpoint. ${ }^{191}$

Yang et al. ${ }^{192}$ experimentally studied the imaging properties, such as the phonon spectrum of the far-field and near-field of graphene/h-BN heterostructures and photon polarization and polarization (Fig. 32); they also compared graphene excitation characteristics based on different substrates. The experimental results showed that the h-BN substrate has small inhibition on

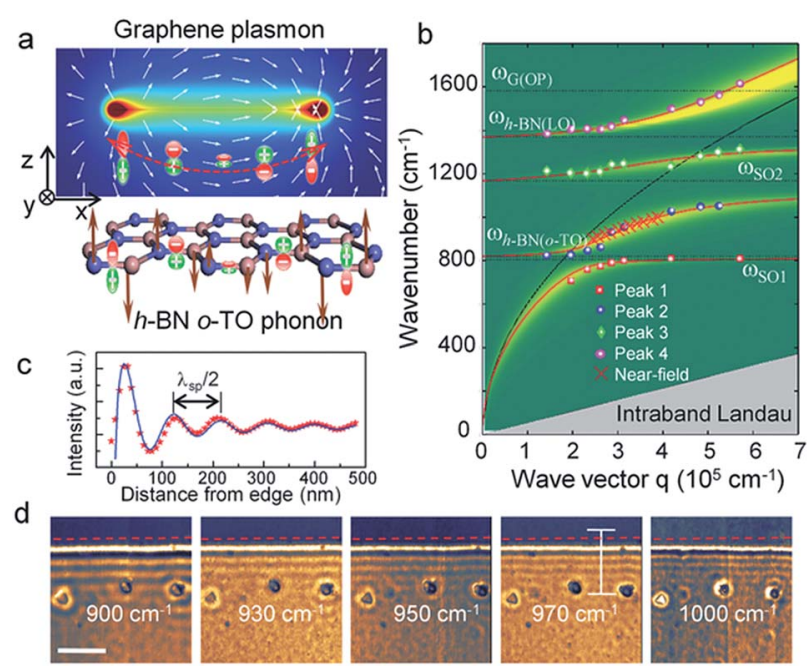

Fig. 32 The coupling mechanism of graphene plasmons and the h-BN optical phonons. (a) The schematic diagram of Graphene plasmon and h-BN o-TO phonon. (b) Plasmon frequencies as a function of wave vector for different hybridized mode peaks. (c) Line profile of s-SNOM optical signals at the excitation wavelength of $970 \mathrm{~cm}^{-1}$. (d) $\mathrm{s}-\mathrm{SNOM}$ images from 2D scan of the tip position near the graphene edge with varied excitation wavelengths. ${ }^{192}$ 
Table 2 Crystallographic information of $\mathrm{h}-\mathrm{BN}$ and graphite ${ }^{193}$

\begin{tabular}{lllll}
\hline & Crystal & $\begin{array}{l}\text { Nearest } \\
\text { neighbour } \\
\text { distance }(\mathrm{nm})\end{array}$ & $\begin{array}{l}\text { Lattice } \\
\text { parameters } \\
(\mathrm{nm})\end{array}$ & $\begin{array}{l}\text { Inter-layer } \\
\text { spacing } \\
(\mathrm{nm})\end{array}$ \\
\hline h-BN & Hexagonal & 0.144 & $\begin{array}{l}a: 0.250 \\
c: 0.666\end{array}$ & 0.333 \\
Graphite & Hexagonal & 0.142 & $\begin{array}{l}a: 0.246 \\
c: 0.670\end{array}$ & 0.335
\end{tabular}

the photoelectric characteristics of graphene, and that h-BN as a graphene substrate has almost no effect on the optical properties of graphene.

4.4.2 Electrical characteristics of graphene/h-BN heterostructures. h-BN with a single crystal has a similar lattice structure to graphite. As an insulating material with a band gap of $5.97 \mathrm{eV}$, h-BN has an atomically flat surface, a very low roughness, no dangling bonds on its surface, and in combination with graphene shows weak van der Waals force, a minimal impact on graphite dilute carrier transport properties, a mismatch of $1.84 \%$ with the graphene lattice ${ }^{72}$ and has no doping effect on graphene. Table 2 presents the differences in their lattice. ${ }^{\mathbf{1 9 3}}$ The mobility in graphene on a boron nitride substrate is up to $10^{5} \mathrm{~cm}^{2} \mathrm{~V}^{-1} \mathrm{~s}^{-1} \cdot{ }^{72,194}$ When its surface flatness suppresses graphene wrinkling, this substrate seems more excellent. Meanwhile, BN has good electrical properties, which can be used for the gate electrode of graphene devices without any loss of functionality of graphene. Furthermore, the optical phonon mode of boron nitride's surface is three times more than the energy of silicon oxide, which means that graphene with a boron nitride substrate may have a better performance under high temperature and a high electric field.

In several experimental preparations of graphene/h-BN, the lattice constants of graphene and h-BN had a 1.84\% difference, and there is a certain angle of rotation between the two hexagonal holes sheet layers, making graphene/h-BN heterostructures form Murray stripes (Fig. 33). ${ }^{\mathbf{7 0 , 1 9 5}}$ This formation of superlattice, results in a lot of new properties, in addition to the zero band gap, Fermi velocity changes, states of jitter of the local density and the superlattice features of the local charge density. ${ }^{196}$

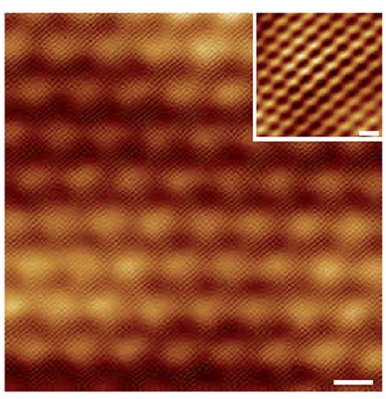

a

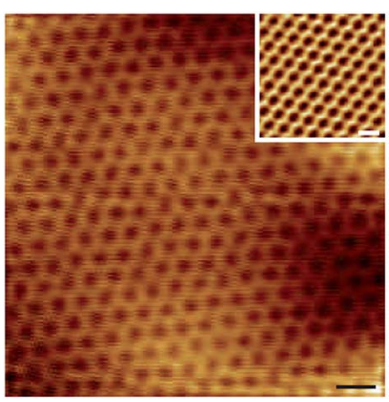

Fig. 33 Real space and Fourier transforms of Moiré patterns with different ratio in (a) and (b). ${ }^{195}$
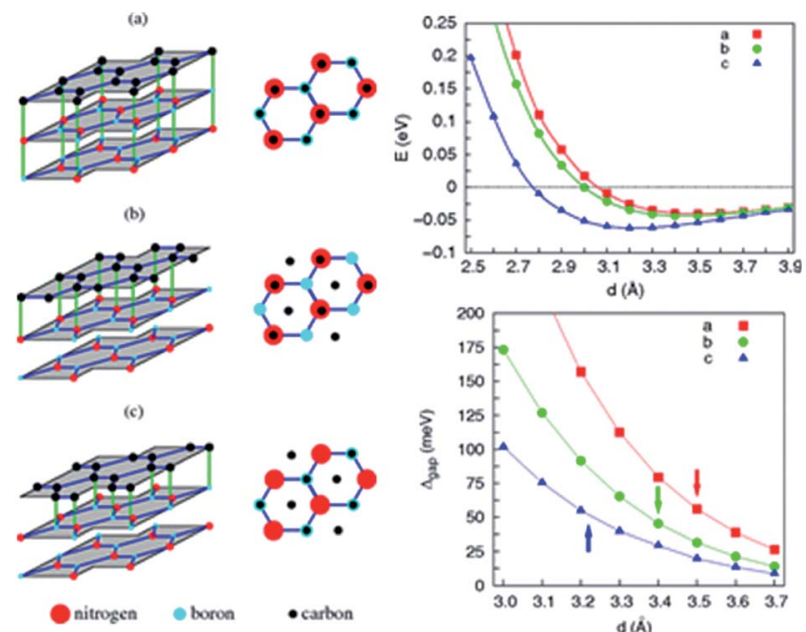

Fig. 34 The three inequivalent orientations of single-layer graphene on a $\mathrm{h}$-BN surface and the total energy $E$ of graphene on a $\mathrm{h}-\mathrm{BN}$ surface for the three manners. ${ }^{71}$

Giovannetti et al.,$^{71}$ in 2007 , proposed the introduction of a band gap (Fig. 34) by depositing the monolayer graphene on a h-BN substrate. With a different stacked manner, the graphene/h-BN heterostructure's band gap varied, and different distances of the layers generated a variation in the band gap. Hüser et al., ${ }^{197}$ by calculations, obtained the electronic properties of graphene/h-BN heterostructures, and found that in the band, some small band gap can be opened. Wang et al. ${ }^{\mathbf{1 9 8}}$ used angular resolution electron spectroscopy, for the first time, to directly measure original and second-level best Dirac band structures (Fig. 35) of a graphene/h-BN heterostructure, and in the original and second-level Dirac cone found up to 100 and $160 \mathrm{meV}$ energy gaps, respectively, which revealed the importance of space inversion symmetry in a heterogeneous structure

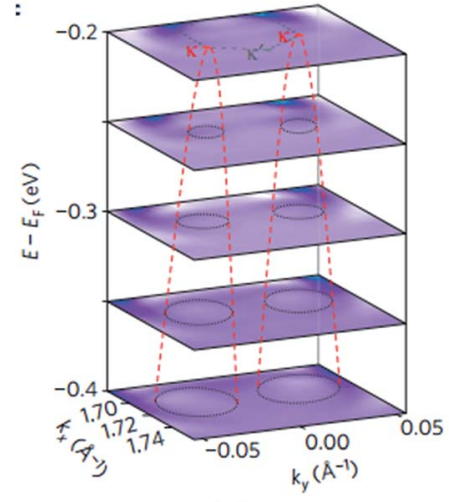

(a)

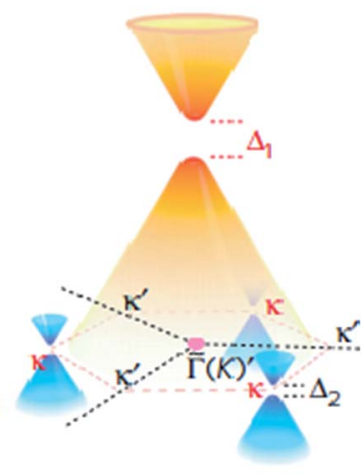

(b)
Fig. 35 (a) Stacking of constant-energy maps of EDC curvature to show the conical dispersion at the two $\kappa$ points. $d 1-d 5$, dispersions along the cuts $\mathrm{d} 1-\mathrm{d} 5$ shown in a1. The white dashed lines are guides for the evolution of the SDCs dispersions. The black arrow indicates the dispersion from the original Dirac cone. (b) Schematic drawing of the band structure in the graphene/h-BN heterostructure, showing the gapped original Dirac point with gapped SDPs at three out of six corners of the SBZ ( $\kappa$ points). ${ }^{198}$ 


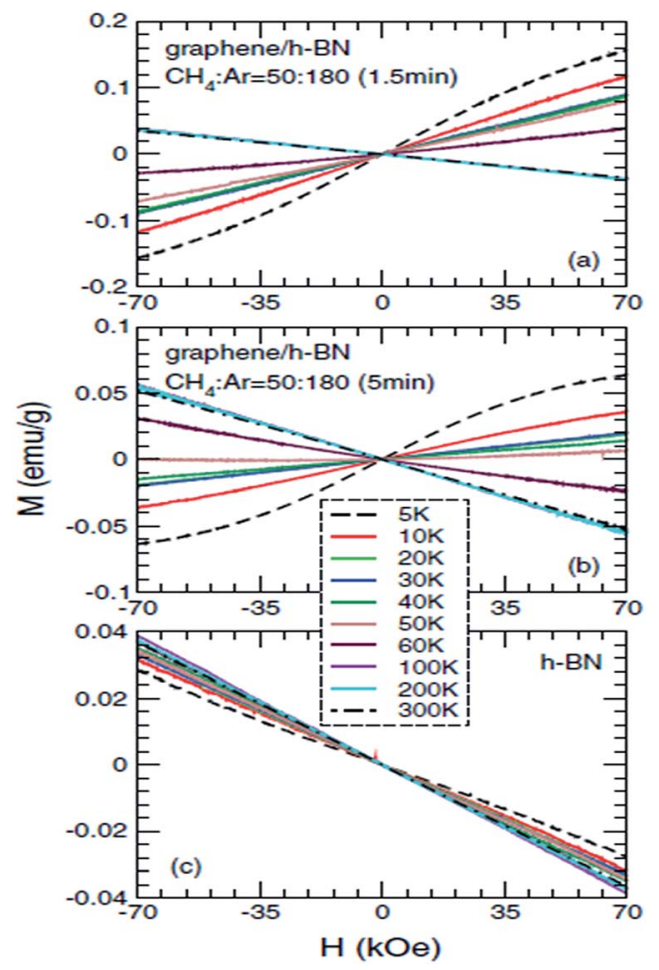

Fig. 36 The measured mass magnetization Mas functions of magnetic fields $\mathrm{H}$ for (a) gra/h-BN sample 1, (b) gra/h-BN sample 2, and (c) h-BN substrate, at different temperatures $T=5,10,20,30,40,50,60,100$, 200 , and $300 \mathrm{~K}^{203}$

and the band modulation capacity of the van der Waals heterostructure.

4.4.3 Magnetic properties of graphene/h-BN heterostructures. Theoretical research on a magnetic $\mathrm{C}$ and h-BN composite system was carried prior to the experimental study. ${ }^{199-202}$ The studies found that the C-BN system appears to have itinerant electron ferromagnetic and antiferromagnetic properties. Ramasubramaniam et al. ${ }^{201}$ in 2011, studied how 2D h-BN graphene nanoislands with mosaic zigzag edges appeared antiferromagnetic by using density functional theory, and found that the antiferromagnetic Nair temperature increased with the density of the graphene unit area in hBN mosaic increasing. Natalia Berseneva et al. ${ }^{202}$ also used this method to carry out calculations on the carbon nanoislands, and found a triangular inlay in h-BN could generate a spin $1 / 2$ magnetic moment. These two models were two models of graphene/h-BN heterostructures composites, and their research provided a theoretical approach for the later research on the magnetic properties of the graphene/h-BN heterostructures composite system.

Ding et al. ${ }^{203}$ in 2011, specifically studied the magnetic properties of graphene/h-BN composites, particularly for abnormal paramagnetic behaviour of the graphene/h-BN composites at low temperature. They proposed an analysis of the low-temperature magnetization curve, and found that the paramagnetic parts $(<20 \mathrm{~K})$ were mainly from the point defects in the sample, and that the angular momentum quantum number $J=1 / 2$. This was the first time that they theoretically explained why $J=l / 2$, and also the first to give $J=1 / 2$ in a graphene system. To study the low-temperature magnetization curve anomalies paramagnetic behaviour (20-50 K), they used several theoretical models to explain this phenomenon, such as the RKKY interaction in the antiferromagnetic spin-glass state, and in the itinerant electron paramagnetic strengthening effect. The experimental results and antiferromagnetic spin-glass theory have a degree of conflict, and only the itinerant electron paramagnetic strengthen theory can be consistent with all of their results. So they speculated that the abnormal paramagnetic behaviour of the graphene/h-BN system at low temperature $(20-50 \mathrm{~K})$ came from the paramagnetic strengthening effect generated by the itinerant electrons from the graphene and h-BN interface or near the interface between (Fig. 36).

\subsection{Potential applications of graphene/h-BN heterostructures}

The introduction of h-BN as a substrate is to solve the graphene in traditional silicon material strain are imposed on the substrate and doping adverse problems. At the same time, formation of a van der Waals' heterostructure of graphene/h-BN increases the mobility of graphene and facilitates making nanometre functional devices, with wide potential application and practical significance.

Field-effect transistor (FET). In 2010, Dean et al. ${ }^{\mathbf{2 0 4}}$ prepared a field-effect transistor from a graphene/h-BN heterostructure for the first time and then tested the device performance (Fig. 37). They used a mechanical stripping method to obtain a single crystal of h-BN on single and double graphene field-effect tubes. The result of the test showed that the mobility of the current carrier reached $60000 \mathrm{~cm}^{2} \mathrm{~V}^{-1} \mathrm{~s}^{-1}$, which was more than three
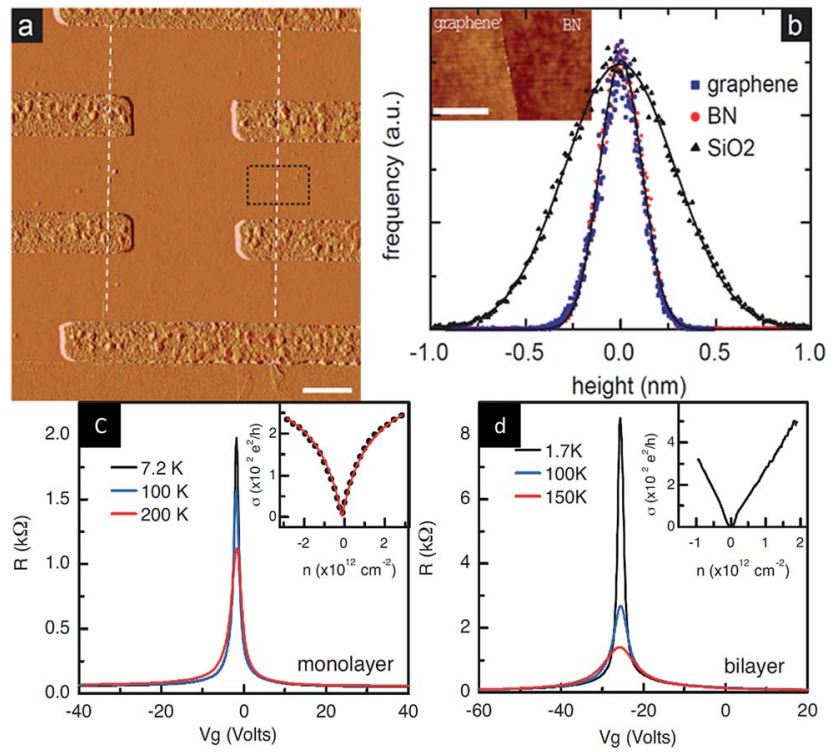

Fig. 37 Graphene/h-BN devices and the test chart. (a) AFM image of graphene/h-BN heterostructure. (b) Histogram of the height distribution measured by AFM for graphene, $\mathrm{h}-\mathrm{BN}$ and $\mathrm{SiO}_{2}$. Resistance versus applied gate voltage for monolayer graphene (c) and bilayer graphene (d) on h-BN. ${ }^{204}$ 

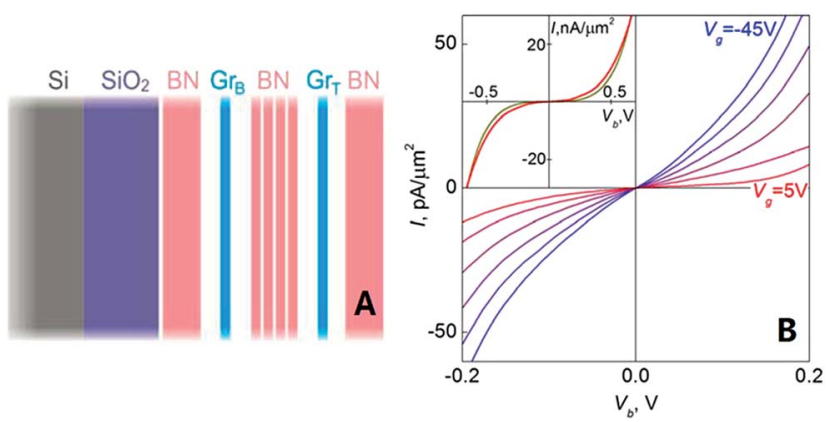

Fig. 38 Schematic of a field-effect tunnelling transistor (A) and (B) tunnelling characteristics for a graphene/h-BN device with $6 \pm 1$ layers of $\mathrm{h}-\mathrm{BN}$ as the tunnel barrier. ${ }^{182}$

times the mobility of graphene, similar to the calculation method for the $\mathrm{SiO}_{2}$ substrate, while the uniformity of charge was less than $7 \times 10^{10} \mathrm{~cm}^{-2}$, three times smaller than silica basal graphene at room temperature. When the temperature was $15 \mathrm{~K}$, the uniformity of charge was $1 \times 10^{9} \mathrm{~cm}^{-2}$, which was similar to suspended graphene. In addition to being an order of magnitude higher than that of the other test results, the roughness, intrinsic doping level, and chemical reactivity were lower.

From then on, more and more similar work was carried out; for instance, in 2011, Decker et al. $;^{70}$ in 2013 and 2014, Lee et al. $;^{205,206}$ also in 2014, Iqbal et al. $;^{207}$ and in 2015, Song et al. $;^{181}$ prepared a FET with graphene/h-BN, which obtained satisfactory results. All of the above, paid witness to the progress of graphene/h-BN van der Waals heterostructures for nanoelectronics applications.

Quantum tunnelling transistor. In 2012, Britnell et al. demonstrated a quantum tunnelling transistor, which was made of a graphene/h-BN heterostructure. In this experiment, they achieved quantum tunnelling from a graphene electrode through h-BN, which was made as an insulating barrier. From the results, they concluded that their tunnelling devices offered a viable method to prepared high speed graphenebased analogue electronics (Fig. 38). ${ }^{182}$
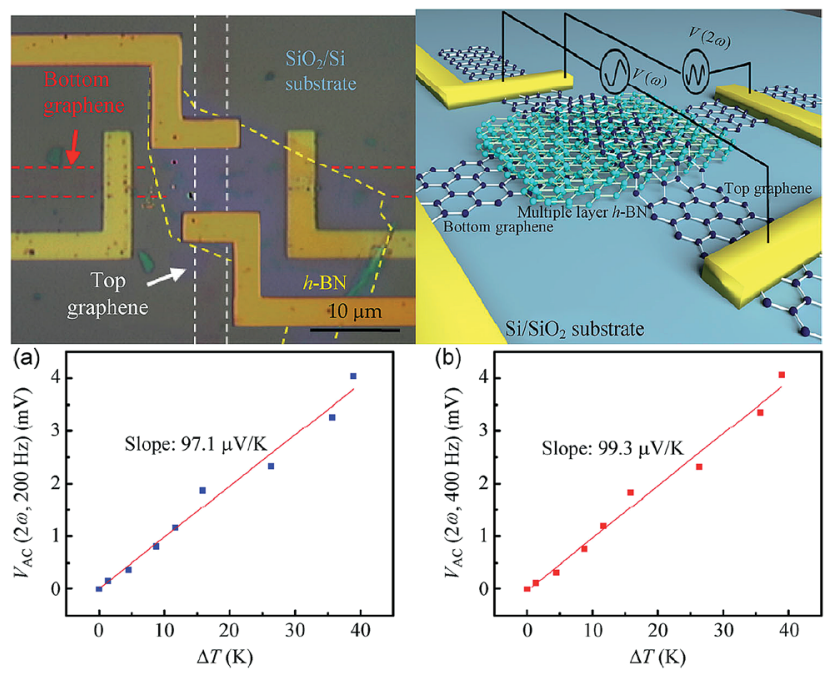

Fig. 39 Schematic and test results of the device. ${ }^{209}$
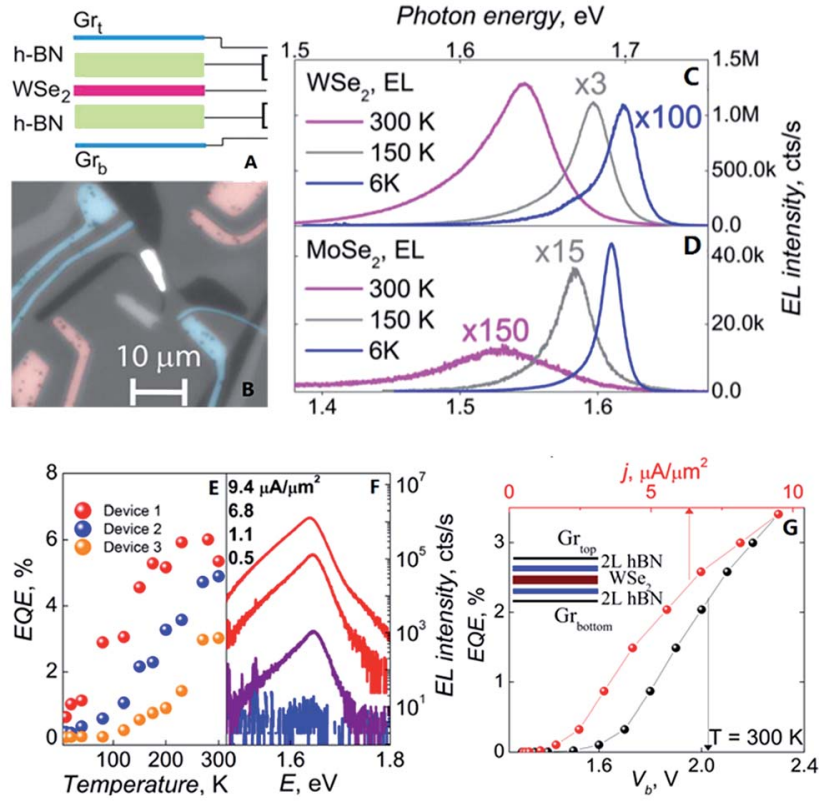

Fig. 40 The flow and test figure of an LED.((A) Schematic of the device architecture. (B) Magnification monochrome image of WSe 2 LEQW device. The change of photon energy under different temperature curve for $\mathrm{WSe}_{2}$ in (C) and $\mathrm{MoSe}_{2}$ in (D). (E) The image of quantum efficiency corresponding to temperature of three LED devices based $\mathrm{WSe}_{2}$. (F) Individual electroluminescence spectra plotted for four different injection current densities for a sample device. (G) The external quantum efficiency plotted against bias voltage and injection current density at $T=300 \mathrm{~K}$ for the same sample device in $(F))^{210}$

Thermoelectric device. In 2014, Chen et al. studied the thermal interface conductance across a graphene/h-BN heterojunction. ${ }^{208}$ Based on the study, they prepared graphene/h-BN heterostructures to measure the thermoelectric transport. ${ }^{209}$ They separated the thermoelectric contribution to the $I-V$

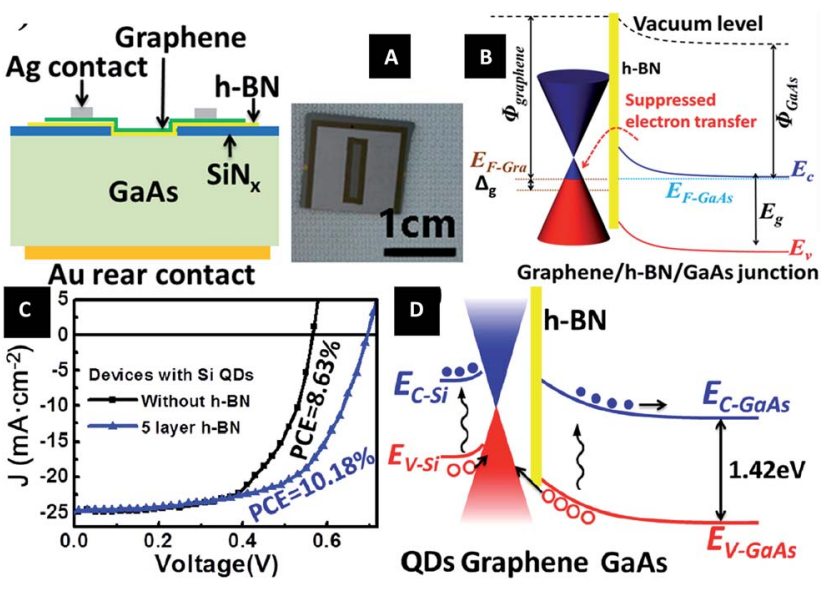

Fig. 41 Graphene/h-BN/GaAs sandwich diode as a solar cell and photodetector. (A) Schematic of the graphene/h-BN/GaAs sandwich device. (B) Electronic band structure of graphene/h-BN/GaAs heterostructure. (C) J-V curves of solar cells based on graphene/GaAs and graphene/h-BN/GaAs heterostructure with Si QDs introduced photoinduced doping. (D) Electronic band structure of the graphene/h-BN/ GaAs solar cell with Si QDs covering on graphene under illumination. ${ }^{212}$ 

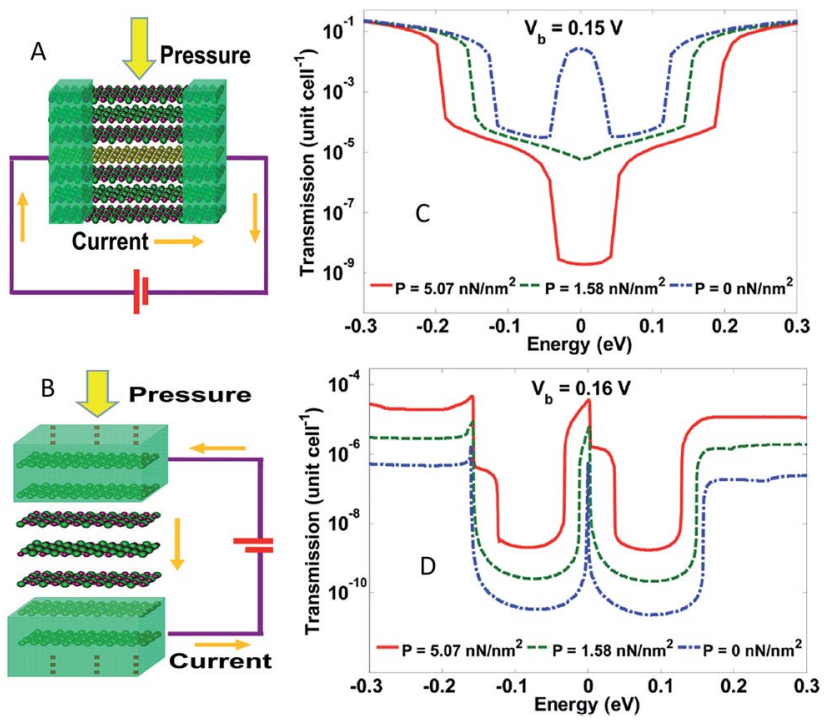

Fig. 42 Schematic of the structures of: (A) an in-plane pressure sensor and (B) a tunnelling pressure sensor. Transmission spectra of: (C) the in-plane sensor and (D) the tunnelling sensor as functions of electron energy with different external pressure. ${ }^{213}$

property of the device structures by using an AC lock-in technique, and, by using Raman spectroscopy, they measured the temperature gradient, so that they could study the thermoelectric transport produced at the interfaces of the heterostructures. Fig. 39 show the thermoelectric voltage and temperature gradient. They ascertained the Seebeck coefficient to be $99.3 \mu \mathrm{V} \mathrm{K}^{-1}$ for the device.

LED. Withers et $a .^{\mathbf{2 1 0}}$ made graphene/h-BN/WSe $/$ h-BN/ graphene heterostructures to prepare an LED in 2015. They used $\mathrm{WSe}_{2}$ light-emitting tunnelling transistors with enhanced brightness at room temperature. They cleaved and exfoliated h$\mathrm{BN}$ onto a $\mathrm{Si} / \mathrm{SiO}_{2}$ substrate; then, a graphene flake was peeled onto an h-BN crystal, and h-BN/graphene/h-BN/WX $/$ h-BN heterostructures were prepared to complete the LED structure. Fig. 40 shows the EQE T-dependence for three $\mathrm{WSe}_{2}$ devices, which shows the characteristic increase with temperature, reaching $5 \%$ at $300 \mathrm{~K}$.

Wang et al. ${ }^{211}$ prepared graphene/h-BN van der Waals lightemitting diodes in 2015. In the two experiments, the heterostructures were made into LEDs, which provides direction for the preparation of nano-optoelectronic devices.

Solar cell. In 2015, Li et al. ${ }^{212}$ designed a graphene/h-BN/GaAs sandwich diode as a solar cell and photodetector. From Fig. 41, we can see that the barrier height of the graphene/GaAs heterojunction was increased from $0.88 \mathrm{eV}$ to $1.02 \mathrm{eV}$, when h-BN was inserted. h-BN enhanced the Fermi level tuning effect, and a power conversion efficiency (PCE) of $10.18 \%$ could be achieved for graphene/h-BN/GaAs, as compared with $8.63 \%$ for the graphene/GaAs structure. The performance of the structure-based photodetector was also improved with the on/off ratio increased.

IPPS and TPS. In 2011, Xu et al. ${ }^{213}$ developed an in-plane pressure sensor (IPPS) and a tunnelling pressure sensor (TPS) made of graphene/h-BN heterostructures. They modelled the responses as a function of external pressure. The results of the tests showed that the current varied by three orders of magnitude as the pressure increased from 0 to $5 \mathrm{nN} \mathrm{nm}^{-2}$. The IPPS current was negatively correlated to pressure, whereas the TPS current exhibited a positive correlation to pressure. IPPS and TPS both had a pressure range of $\sim 5 \mathrm{GPa}$, which was bigger than conventional MEMS pressure sensors (Fig. 42). The results pointed to the direction towards realizing viable, atomic scale graphene-based sensors for pressure measurements.

Combining boron nitride with graphene offers multidimensional application opportunities in many fields: from the perspective of transport doping, from both horizontal and vertical components. People can conduct researches on physic based on boron nitride excellent substrate effect, and also can do researches on electronics to use boron nitride wide band gap. Therefore, there is still a hope to see good research into the aspects of mixing graphite and boron nitride.

\section{Summaries and outlook}

For logic devices of graphene/h-BN heterostructures, although a lot of theories and experiments prove that graphene based on $\mathrm{h}$ $\mathrm{BN}$ has excellent optical characteristics, because of the zero bandgap properties of graphene, it is difficult to prepare a highperformance logic circuit as a direct field-effect transistor. Therefore, the band of $2 \mathrm{D}$ carbon-based material has a very important role in the regulation of optoelectronic device applications. For example, continuous research of graphene/h-BN heterostructures in the far infrared, the visible region and in the ultraviolet region can provide a basis for the preparation of applications of photovoltaic devices. Accounting for the different absorptions of graphene and h-BN in light regions, it is certain that a composite system of graphene/h-BN heterostructures has great use in the visible region, such as photoluminescence. On the other hand, regulation of the energy band engineering can be achieved by designing $2 \mathrm{D}$ heterostructure materials in combination with dielectric shielding. How the graphene layer of the graphene/h-BN heterostructure and the h-BN layer are bonded, and what is the force between the electrons will be revealed in the future. We believe that practical applications of heterostructure graphene/h-BN will greatly expand because of the unique optoelectronic properties of this structure.

For graphene, due to its characteristics it is useful for optoelectronic devices, energy storage, and other aspects of mechanical reinforcement, and in line with its potential, the basic applied research in new 2D materials based on graphene has made great progress. When combined with other new $2 \mathrm{D}$ materials, by using 2D layered materials with different chemical bonds and the formation of different physical and chemical properties of new lattice structures, it should have even greater applications. For example: biomedical devices, photodetectors, chemical catalysis, clean energy generation and storage, surface-enhanced Raman scattering and so on.

Perhaps one of the most meaningful aspects of the research into graphene is that graphene has caused researchers to explore other 2D materials, which is a break from the long-term norm of studying 3D materials. At least in the history of science 
in materials, before graphene occurred, there was no one focusing on studying $2 \mathrm{D}$ materials. Therefore, the heuristic value of graphene is far greater than just the value of its own excellent performance. For instance, by combining the same or similar 2D structures of several different materials, the resulting new composite material have a lot of new characteristics that are exciting the scientific community, and hence the occurrence of $2 \mathrm{D}$ composite materials can help make the properties of the new materials more diversified.

\section{Acknowledgements}

This work was supported by National Natural Science Foundation of China (Grant No. 11374353, 91436102 and 11274149), National Basic Research Program of China (Grant number 2016YFA02008000), and the Program of Liaoning Key Laboratory of Semiconductor Light Emitting and Photocatalytic Materials.

\section{References}

1 A. K. Geim and K. S. Novoselov, Nat. Mater., 2007, 6, 183191.

2 T. Ohta, A. Bostwick, T. Seyller, K. Horn and E. Rotenberg, Science, 2006, 313, 951-954.

3 K. S. Novoselov, A. K. Geim, S. V. Morozov, D. Jiang, Y. Zhang, S. V. Dubonos, I. V. Grigorieva and A. A. Firsov, Science, 2004, 306, 666-669.

4 J. Chen, Nature, 2012, 487, 77-81.

5 X. Geng, L. Niu, Z. Xing, R. Song, G. Liu, M. Sun, G. Cheng, H. Zhong, Z. Liu and Z. Zhang, Adv. Mater., 2010, 22, 638642.

6 A. K. Geim, Angew. Chem., 2011, 50, 6966-6985.

7 R. Dagani, Nat. Nanotechnol., 2007, 2, 605-615.

8 Y. B. Tang, C. S. Lee, Z. H. Chen, G. D. Yuan, Z. H. Kang, L. B. Luo, H. S. Song, Y. Liu, Z. B. He and W. J. Zhang, Nano Lett., 2009, 9, 1374-1377.

9 C. Lee, X. Wei, J. W. Kysar and J. Hone, Science, 2008, 321, 385-388.

10 K. I. Bolotin, K. J. Sikes, Z. Jiang, M. Klima, G. Fudenberg, J. Hone, P. Kim and H. L. Stormer, Solid State Commun., 2008, 146, 351-355.

11 B. H. Hong, Nature, 2009, 457, 706-710.

12 A. A. Balandin, Nat. Mater., 2011, 10, 569-581.

13 L. A. Falkovsky, Phys. Lett. A, 2008, 372, 5189-5192.

14 S. Iijima, Nature, 1991, 354, 56-58.

15 D. S. Bethune, C. H. Klang, M. S. D. Vries, G. Gorman, R. Savoy, J. Vazquez and R. Beyers, Nature, 1993, 363, 605-607.

16 S. Iijima and T. Ichihashi, Nature, 1993, 364, 603-605.

17 L. Guan, Nano Lett., 2008, 8, 459-462.

18 Q. Wen, R. Zhang, W. Qian, Y. Wang, P. Tan, J. Nie and F. Wei, Chem. Mater., 2010, 22, 1294-1296.

19 S. Iijima, P. M. Ajayan and T. Ichihashi, Phys. Rev. Lett., 1992, 69, 3100-3103.

20 D. Galpaya, M. Wang, G. George and N. Motta, J. Appl. Phys., 2001, 116, 053510-053518.
21 R. Saito, M. Fujita, G. Dresselhaus and M. S. Dresselhaus, Appl. Phys. Lett., 1992, 60, 2204-2206.

22 N. Hamada, S. Sawada and A. Oshiyama, Phys. Rev. Lett., 1992, 68, 1579-1581.

23 M. M. J. Treacy, T. W. Ebbesen and J. M. Gibson, Nature, 1996, 381, 678-680.

24 O. Lourie, D. M. Cox and H. D. Wagner, Phys. Rev. Lett., 1998, 81, 1638-1641.

25 M. Ritschel, M. Uhlemann, O. Gutfleisch, A. Leonhardt, A. Graff, C. Täschner and J. Fink, Appl. Phys. Lett., 2002, 80, 2985-2987.

26 J. Kong, N. R. Franklin, C. Zhou, M. G. Chapline, S. Peng, K. Cho and H. Dai, Science, 2000, 287, 622-625.

27 P. L. Mceuen, M. S. Fuhrer and H. Park, IEEE Trans. Nanotechnol., 2002, 1, 78-85.

28 J. Appenzeller, R. Martel, V. Derycke, M. Radosavljevi, S. Wind, D. Neumayer and P. Avouris, Microelectron. Eng., 2002, 64, 391-397.

29 W. A. Deheer, W. S. Bacsa, A. Châtelain, T. Gerfin, R. Humphreybaker, L. Forro and D. Ugarte, Science, 1995, 268, 845-847.

30 F. Bommeli, L. Degiorgi, P. Wachter, W. S. Bacsa, W. A. D. Heer and L. Forro, Synth. Met., 1997, 86, 2307-2308.

31 X. Gao, Z. Zhou, Y. Zhao, S. Nagase, S. B. Zhang and Z. Chen, J. Phys. Chem. C, 2008, 112, 12677-12682.

32 P. B. Wheelock, B. C. Cook, J. L. Harringa and A. M. Russell, J. Mater. Sci., 2004, 39, 343-347.

33 C. Lorrette, P. Weisbecker, S. Jacques, R. Pailler and J. M. Goyhénèche, J. Eur. Ceram. Soc., 2007, 27, 2737-2743.

34 G. E. Lei, J. Yang and T. Qiu, Electron. Compon. Mater., 2008, 27, 22-29.

35 K. Watanabe, T. Taniguchi and H. Kanda, Nat. Mater., 2004, 3, 404-409.

36 Y. Kubota, K. Watanabe, O. Tsuda and T. Taniguchi, Science, 2007, 38, 932-934.

37 D. Golberg, Y. Bando, Y. Huang, T. Terao, M. Mitome, C. Tang and C. Zhi, ACS Nano, 2010, 4, 2979-2993.

38 D. Pacilé, J. C. Meyer, Ç. Ö. Girit and A. Zettl, Appl. Phys. Lett., 2008, 92, 1331071-1331073.

39 C. Lee, Q. Li, W. Kalb, X. Z. Liu, H. Berger, R. W. Carpick and J. Hone, Science, 2010, 328, 76-80.

40 A. Nagashima, N. Tejima, Y. Gamou, T. Kawai and C. Oshima, Phys. Rev. Lett., 1995, 75, 3918-3921.

41 K. S. Novoselov, D. Jiang, F. Schedin, T. J. Booth, V. V. Khotkevich, S. V. Morozov and A. K. Geim, Proc. Natl. Acad. Sci. U. S. A., 2005, 102, 10451-10453.

42 T. P. Crane and B. P. Cowan, Phys. Rev. B: Condens. Matter Mater. Phys., 2000, 62, 11359-11362.

43 M. Miller and F. J. Owens, Solid State Commun., 2011, 151, 1001-1003.

44 E. K. Sichel, R. E. Miller, M. S. Abrahams and C. J. Buiocchi, Phys. Rev. B: Condens. Matter Mater. Phys., 1976, 13, 4607-4611.

45 C. H. Henager Jr and W. T. Pawlewicz, Appl. Opt., 1993, 32, 91-101.

46 J. Ravichandran, A. G. Manoj, J. Liu, I. Manna and D. L. Carroll, Nanotechnology, 2008, 19, 085712. 
47 S. Hao, G. Zhou, W. Duan, J. Wu and B. L. Gu, J. Am. Chem. Soc., 2006, 128, 8453-8458.

48 G. J. Zhang, M. Ando, T. Ohji and S. Kanzaki, Int. J. Appl. Ceram. Technol., 2005, 2, 162-171.

49 M. S. Jin and N. O. Kim, J. Electr. Eng. Technol., 2010, 5, 637639.

50 Y. Zhang, X. He, J. Han and S. Du, J. Mater. Process. Technol., 2001, 116, 161-164.

51 C. Harrison, S. Weaver, C. Bertelsen, E. Burgett, N. Hertel and E. Grulke, J. Appl. Polym. Sci., 2008, 109, 2529-2538.

52 J. A. Hanigofsky, K. L. More, W. J. Lackey, W. Y. Lee and G. B. Freeman, J. Am. Ceram. Soc., 1991, 74, 301-305.

53 R. Naslain, O. Dugne, A. Guette, J. Sevely, C. R. Brosse, J. P. Rocher and J. Cotteret, J. Am. Ceram. Soc., 1991, 74, 2482-2488.

54 T. Mashoff, M. Pratzer, V. Geringer, T. J. Echtermeyer, M. C. Lemme, M. Liebmann and M. Morgenstern, Nano Lett., 2009, 10, 461-465.

55 J. C. Hamilton and J. M. Blakely, Surf. Sci., 1980, 91, 199217.

56 A. M. Shikin, G. V. Prudnikova, V. K. Adamchuk, F. Moresco and K. H. Rieder, Phys. Rev. B: Condens. Matter Mater. Phys, 2000, 62, 13202-13208.

57 R. Rosei, M. De Crescenzi, F. Sette, C. Quaresima, A. Savoia and P. Perfetti, Phys. Rev. B: Condens. Matter Mater. Phys, 1983, 28, 1161-1164.

58 A. M. Shikin, D. Farías and K. H. Rieder, EPL, 2007, 44, 4449.

59 Y. S. Dedkov, A. M. Shikin, V. K. Adamchuk, S. L. Molodtsov, C. Laubschat, A. Bauer and G. Kaindl, Phys. Rev. B: Condens. Matter Mater. Phys., 2001, 64, 0354051-0354056.

60 T. Brugger, S. Günther, B. Wang, J. H. Dil, M. L. Bocquet, J. Osterwalder, J. Wintterlin and T. Greber, Phys. Rev. B: Condens. Matter Mater. Phys., 2009, 79, 0454071-0454076.

61 W. Moritz, B. Wang, M. L. Bocquet, T. Brugger, T. Greber, J. Wintterlin and S. Günther, Phys. Rev. Lett., 2010, 104, 1361021-1361024.

62 T. A. Land, T. Michely, R. J. Behm, J. C. Hemminger and G. Comsa, Surf. Sci., 1992, 264, 261-270.

63 D. E. Starr, E. M. Pazhetnov, A. I. Stadnichenko, A. I. Boronin and S. K. Shaikhutdinov, Surf. Sci., 2006, 600, 2688-2695.

64 J. H. Chen, C. Jang, S. Xiao, M. Ishigami and M. S. Fuhrer, Nat. Nanotechnol., 2008, 3, 206-209.

65 S. L. Dong, C. Riedl, B. Krauss, K. V. Klitzing, U. Starke and J. H. Smet, Nano Lett., 2008, 8, 4320-4325.

66 V. Geringer, M. Liebmann, T. Echtermeyer, S. Runte, M. Schmidt, R. Rückamp, M. C. Lemme and M. Morgenstern, Phys. Rev. Lett., 2008, 102, 076102.

67 I. Forbeaux, J. M. Themlin and J. M. Debever, Phys. Rev. B, 1998, 58, 16396.

68 T. G. Mendes-De-Sa, A. M. Goncalves, M. J. Matos, P. M. Coelho, R. Magalhaes-Paniago and R. G. Lacerda, Nanotechnology, 2012, 23, 475602.

69 J. Hass, F. Varchon, J. E. Millán-Otoya, M. Sprinkle, N. Sharma, W. A. de Heer, C. Berger, P. N. First,
L. Magaud and E. H. Conrad, Phys. Rev. Lett., 2008, 100, 125504.

70 R. Decker, Y. Wang, V. W. Brar, W. Regan, H. Z. Tsai, Q. Wu, W. Gannett, A. Zettl and M. F. Crommie, Nano Lett., 2011, 11, 2291-2295.

71 G. Giovannetti, P. A. Khomyakov, G. Brocks, P. J. Kelly and J. V. D. Brink, Phys. Rev. B: Condens. Matter Mater. Phys., 2007, 76, 3009-3014.

72 C. R. Dean, A. F. Young, I. Meric, C. Lee, L. Wang, S. Sorgenfrei, K. Watanabe, T. Taniguchi, P. Kim and K. L. Shepard, Nat. Nanotechnol., 2010, 5, 722.

73 L. A. Ponomarenko, A. K. Geim, A. A. Zhukov, R. Jalil, S. V. Morozov, K. S. Novoselov, I. V. Grigorieva, E. H. Hill, V. V. Cheianov and V. I. Fal'Ko, Nat. Phys., 2011, 7, 958-961.

74 L. Britnell, R. V. Gorbachev, R. Jalil, B. D. Belle, F. Schedin, A. Mishchenko, T. Georgiou, M. I. Katsnelson, L. Eaves and S. V. Morozov, Science, 2012, 335, 947-950.

75 S. J. Haigh, A. Gholinia, R. Jalil, S. Romani, L. Britnell, D. C. Elias, K. S. Novoselov, L. A. Ponomarenko, A. K. Geim and R. Gorbachev, Nat. Mater., 2012, 11, 764.

76 C. Dean, A. F. Young, L. Wang, I. Meric, G. H. Lee, K. Watanabe, T. Taniguchi, K. Shepard, P. Kim and J. Hone, Solid State Commun., 2012, 152, 1275-1282.

77 L. A. Ponomarenko, R. V. Gorbachev, G. L. Yu, D. C. Elias, R. Jalil, A. A. Patel, A. Mishchenko, A. S. Mayorov, C. R. Woods and J. R. Wallbank, Nature, 2013, 497, 594-597.

78 C. R. Dean, L. Wang, P. Maher, C. Forsythe, F. Ghahari, Y. Gao, J. Katoch, M. Ishigami, P. Moon and M. Koshino, Nature, 2013, 497, 598-602.

79 B. Partoens and F. M. Peeters, Phys. Rev. B: Condens. Matter Mater. Phys., 2006, 74, 07540401-07540411.

80 P. Ma, Z. Jin, J. N. Guo, H. L. Pan, X. Y. Liu, T. C. Ye, Y. P. Jia, L. W. Guo and X. L. Chen, Sci. Bull., 2012, 57, 2401-2404.

81 M. Wu, X. Wu, Y. Gao and X. C. Zeng, Appl. Phys. Lett., 2009, 94, 2231111-2231113.

82 B. Biel, F. Triozon, X. Blase and S. Roche, Nano Lett., 2009, 9, 2725-2729.

83 L. Y. Jiao, L. Zhang, X. R. Wang, G. Diankov and H. J. Dai, Nature, 2012, 458, 877-880.

84 P. W. Sutter, J. I. Flege and E. A. Sutter, Nat. Mater., 2008, 7, 406-411.

85 C. Berger, Z. Song, X. Li, X. Wu, N. Brown, C. Naud, D. Mayou, T. Li, J. Hass and A. N. Marchenkov, Science, 2006, 312, 1191-1196.

86 H. Wang and G. Yu, Adv. Mater., 2016, 28, 4956-4975.

87 C. Gómez-Navarro, M. Burghard and K. Kern, Nano Lett., 2008, 8, 2045-2049.

88 M. Poot, Appl. Phys. Lett., 2008, 92, 0631111-0631113.

89 D. Yoon, Y. W. Son and H. Cheong, Nano Lett., 2011, 11, 3227-3231.

90 J. H. Seol, I. Jo, A. L. Moore, L. Lindsay, Z. H. Aitken, M. T. Pettes, X. Li, Z. Yao, R. Huang and D. Broido, Science, 2010, 328, 213.

91 Y. Xu, X. Chen, B. L. Gu and W. Duan, Appl. Phys. Lett., 2009, 95, 2331161-2331164.

92 Z. H. Ni, H. M. Wang, J. Kasim, H. M. Fan, T. Yu, Y. H. Wu, Y. P. Feng and Z. X. Shen, Nano Lett., 2007, 7, 2758. 
93 R. R. Nair, P. Blake, A. N. Grigorenko, K. S. Novoselov, T. J. Booth, T. Stauber, N. M. R. Peres and A. K. Geim, Science, 2008, 320, 1308.

94 A. B. Kuzmenko, H. E. Van, F. Carbone and d. M. D. Van, Phys. Rev. Lett., 2008, 100, 117401.

95 J. P. Salvetat, J. M. Bonard, L. Forró, R. Bacsa and T. Stöckli, 1998. 467-480.

96 A. H. C. Neto, Vacuum, 2009, 83, 1248-1252.

97 M. Wu, C. Cao and J. Z. Jiang, Nanotechnology, 2010, 21, 505202-505207.

98 A. Lherbier, X. Blase, Y. M. Niquet, F. Triozon and S. Roche, Phys. Rev. Lett., 2008, 101, 036808.

99 A. Varykhalov, M. R. Scholz, T. K. Kim and O. Rader, Phys. Rev. B: Condens. Matter Mater. Phys., 2010, 82, 121101.

100 V. M. Pereira, A. H. C. Neto and N. M. R. Peres, Phys. Rev. B: Condens. Matter Mater. Phys., 2008, 80, 1132-1136.

101 E. V. Castro, K. S. Novoselov, S. V. Morozov, N. M. Peres, J. M. dos Santos, J. Nilsson, F. Guinea, A. K. Geim and A. H. Neto, Phys. Rev. Lett., 2007, 99, 216802.

102 S. Y. Zhou, G. H. Gweon, A. V. Fedorov, P. N. First, W. A. de Heer, D. H. Lee, F. Guinea, A. H. Castro Neto and A. Lanzara, Nat. Mater., 2007, 6, 770-775.

103 A. Mattausch and O. Pankratov, Phys. Rev. Lett., 2007, 99, 076802.

104 P. Shemella and S. K. Nayak, Appl. Phys. Lett., 2009, 94, 0321011-0321013.

105 H. X. Yang, A. Hallal, D. Terrade, X. Waintal, S. Roche and M. Chshiev, Phys. Rev. Lett., 2013, 110, 046603.

106 K. S. Novoselov, A. K. Geim, S. V. Morozov, D. Jiang, M. I. Katsnelson, I. V. Grigorieva, S. V. Dubonos and A. A. Firsov, Nature, 2005, 438, 197.

107 Y. Zhang, Y. W. Tan, H. L. Stormer and P. Kim, Nature, 2005, 438, 201.

108 N. Tombros, C. Jozsa, M. Popinciuc, H. T. Jonkman and B. J. V. Wees, Nature, 2007, 448, 571-574.

109 S. Cho, Y. F. Chen and M. S. Fuhrer, Appl. Phys. Lett., 2007, 91, 1231051-1231053.

110 Y. Wang, Y. Huang, Y. Song, X. Zhang, Y. Ma, J. Liang and Y. Chen, Nano Lett., 2009, 9, 220.

111 M. Sepioni, R. R. Nair, S. Rablen, J. Narayanan, F. Tuna, R. Winpenny, A. K. Geim and I. V. Grigorieva, Phys. Rev. Lett., 2010, 105, 207205.

112 G. Gómez-Santos and T. Stauber, Phys. Rev. Lett., 2011, 106, 045504 .

113 A. C. Ferrari, J. C. Meyer, V. Scardaci, C. Casiraghi, M. Lazzeri, F. Mauri, S. Piscanec, D. Jiang, K. S. Novoselov and S. Roth, Phys. Rev. Lett., 2006, 97, 187401.

114 M. S. Dresselhaus, G. Dresselhaus and M. Hofmann, Adv. Phys., 2011, 366, 231.

115 S. Berciaud, S. Ryu, L. E. Brus and T. F. Heinz, Nano Lett., 2009, 9, 346.

116 Y. Wang, D. C. Alsmeyer and R. L. Mccreery, Chem. Mater., 1990, 2, 557-563.

117 Z. Liu, D. He, Y. Wang, H. Wu, J. Wang and H. Wang, Sol. Energy Mater. Sol. Cells, 2010, 94, 2148-2153.

118 M. Topsakal, E. Aktürk and S. Ciraci, Phys. Rev. B: Condens. Matter Mater. Phys., 2009, 79, 11544201-11544211.
119 A. Bhattacharya, S. Bhattacharya and G. P. Das, Phys. Rev. B: Condens. Matter Mater. Phys., 2011, 84, 2789.

120 M. Q. Le, J Mech. Sci. Technol., 2014, 28, 4173-4178.

121 B. Mortazavi and G. Cuniberti, RSC Adv., 2014, 4, 19137.

122 M. Corso, W. Auwaerter, M. Muntwiler, A. Tamai, T. Greber and J. Osterwalder, Science, 2004, 303, 217-220.

123 R. Laskowski, P. Blaha, T. Gallauner and K. Schwarz, Phys. Rev. Lett., 2007, 98, 106802.

124 G. B. Grad, P. Blaha, K. Schwarz, W. Auwärter and T. Greber, Phys. Rev. B: Condens. Matter Mater. Phys., 2003, 68, 085404.

125 A. A. Tonkikh, E. N. Voloshina, P. Werner, H. Blumtritt, B. Senkovskiy, G. Güntherodt, S. S. Parkin and Y. S. Dedkov, Sci. Rep., 2016, 6, 23547.

126 W. Auwärter, M. Muntwiler, J. Osterwalder and T. Greber, Surf. Sci., 2003, 545, L735.

127 M. N. Huda and L. Kleinman, Phys. Rev. B: Condens. Matter Mater. Phys., 2006, 74, 075418.

128 A. Nagashima, N. Tejima, Y. Gamou, T. Kawai and C. Oshima, Phys. Rev. B: Condens. Matter Mater. Phys., 1995, 51, 4606-4613.

129 W. Q. Han, L. Wu, Y. Zhu and K. Watanabe, Appl. Phys. Lett., 2008, 93, 223103.

130 C. Jin, F. Lin, K. Suenaga and S. Iijima, Phys. Rev. Lett., 2009, 102, 195505.

131 R. Gao, L. Yin, C. Wang, Y. Qi, N. Lun, L. Zhang, Y. X. Liu, L. Kang and X. Wang, J. Phys. Chem. C, 2009, 113, 1516015165.

132 J. C. Meyer, A. Chuvilin, G. Algara-Siller, J. Biskupek and U. Kaiser, Nano Lett., 2009, 9, 2683.

133 L. Song, L. Ci, H. Lu, P. B. Sorokin, C. Jin, J. Ni, A. G. Kvashnin, D. G. Kvashnin, J. Lou and B. I. Yakobson, Nano Lett., 2010, 10, 3209.

134 A. Nag, K. Raidongia, K. P. Hembram, R. Datta, U. V. Waghmare and C. N. Rao, ACS Nano, 2010, 4, 15391544.

135 Y. Lin, T. V. Williams, T. B. Xu, W. Cao, H. E. Elsayedali and J. W. Connell, J. Phys. Chem. C, 2011, 115, 2679-2685.

136 J. H. Warner, M. H. Rümmeli, A. Bachmatiuk and B. Büchner, ACS Nano, 2010, 4, 1299-1304.

137 Y. Lin, T. V. Williams and J. W. Connell, J. Phys. Chem. Lett., 2009, 1, 277-283.

138 C. Zhi, Y. Bando, C. Tang, H. Kuwahara and D. Golberg, Adv. Mater., 2009, 21, 2889-2893.

139 A. Goriachko, Y. He, A. Marcus Knapp, H. Over, M. Corso, T. Brugger, S. Berner, A. Juerg Osterwalder and T. Greber, Langmuir, 2007, 23, 2928-2931.

140 L. Boldrin, F. Scarpa, R. Chowdhury and S. Adhikari, Nanotechnology, 2011, 22, 505702.

141 R. C. Andrew, R. E. Mapasha, A. M. Ukpong and N. Chetty, Phys. Rev. B: Condens. Matter Mater. Phys., 2012, 85, 777782.

142 H. Zhou, J. Zhu, Z. Liu, Z. Yan, X. Fan, J. Lin, G. Wang, Q. Yan, T. Yu and P. M. Ajayan, Nano Res., 2014, 7, 12321240.

143 I. Jo, M. T. Pettes, J. Kim, K. Watanabe, T. Taniguchi, Z. Yao and L. Shi, Nano Lett., 2013, 13, 550-554. 
144 C. C. Chen, Z. Li, L. Shi and S. B. Cronin, Nano Res., 2015, 8, 666-672.

145 W. Zhou, S. Qi, Q. An, H. Zhao and N. Liu, Mater. Res. Bull., 2007, 42, 1863-1873.

146 V. Barone and J. E. Peralta, Nano Lett., 2008, 8, 2210-2214.

147 R. Q. Wu, L. Liu, G. W. Peng and Y. P. Feng, Appl. Phys. Lett., 2005, 86, 122510-122513.

148 R. Q. Wu, G. W. Peng, L. Liu and Y. P. Feng, J. Phys.: Condens. Matter, 2006, 18, 569-576.

149 M. S. Si and D. S. Xue, Phys. Rev. B: Condens. Matter Mater. Phys., 2007, 75, 1934091-1934094.

150 L. Lai, J. Lu, L. Wang, G. Luo, J. Zhou, R. Qin, Z. Gao and W. N. Mei, J. Phys. Chem. C, 2010, 113, 2273-2276.

151 F. Zheng, G. Zhou, Z. Liu, J. Wu, W. Duan, B. L. Gu and S. B. Zhang, Phys. Rev. B: Condens. Matter Mater. Phys., 2008, 78, 2054151-2054155.

152 R. V. Gorbachev, I. Riaz, R. R. Nair, R. Jalil, L. Britnell, B. D. Belle, E. W. Hill, K. S. Novoselov, K. Watanabe and T. Taniguchi, Small, 2011, 7, 465.

153 M. I. Katsnelson, K. S. Novoselov and A. K. Geim, Nat. Phys., 2006, 2, 620-625.

154 B. Huard, J. A. Sulpizio, N. Stander, K. Todd, B. Yang and D. Goldhabergordon, Phys. Rev. Lett., 2007, 98, 236803.

155 D. C. Elias, R. R. Nair, T. M. G. Mohiuddin, S. V. Morozov, P. Blake, M. P. Halsall, A. C. Ferrari, D. W. Boukhvalov, M. I. Katsnelson and A. K. Geim, Science, 2009, 323, 610613.

156 L. Liu and Z. Shen, Appl. Phys. Lett., 2009, 95, 252104.

157 D. H. Oh, B. G. Shin and J. R. Ahn, Appl. Phys. Lett., 2010, 96, 951.

158 Y. Zhang, T. T. Tang, C. Girit, Z. Hao, M. C. Martin, A. Zettl, M. F. Crommie, Y. R. Shen and F. Wang, Nature, 2009, 459, 820.

159 Y. Guo, W. Guo and C. Chen, Appl. Phys. Lett., 2008, 92, 243101-243103.

160 C. Oshima, A. Itoh, E. Rokuta, T. Tanaka, K. Yamashita and T. Sakurai, Solid State Commun., 2000, 116, 37-40.

161 J. Stewart, Phys. Rev. B: Condens. Matter Mater. Phys., 2010, 81, 2149.

162 C. Bjelkevig, Z. Mi, J. Xiao, P. A. Dowben, L. Wang, W. N. Mei and J. A. Kelber, J. Phys.: Condens. Matter, 2010, 22, 302002 .

163 S. Tang, G. Ding, X. Xie, J. Chen, C. Wang, X. Ding, F. Huang, W. Lu and M. Jiang, Carbon, 2012, 50, 329-331.

164 S. Tang, H. Wang, H. S. Wang, Q. Sun, X. Zhang, C. Cong, H. Xie, X. Liu, X. Zhou and F. Huang, Nat. Commun., 2015, 6, 6499.

165 X. Zhong, Y. K. Yap, R. Pandey and S. P. Karna, Phys. Rev. B: Condens. Matter Mater. Phys., 2011, 83, 193403.

166 Y. Fan, M. Zhao, Z. Wang, X. Zhang and H. Zhang, Appl. Phys. Lett., 2011, 98, 083103.

167 E. Kan, H. Ren, F. Wu, Z. Li, R. Lu, C. Xiao, K. Deng and J. Yang, J. Phys. Chem. C, 2016, 116, 3142-3146.

168 G. Kresse and J. Hafner, Phys. Rev. B: Condens. Matter Mater. Phys., 1993, 48, 558-561.
169 G. Gao, G. Wei, E. Cannuccia, J. Tahatijerina, L. Balicas, A. Mathkar, T. N. Narayanan, L. Zhen, B. K. Gupta and J. Peng, Nano Lett., 2012, 12, 3518-3525.

170 P. J. Zomer, S. P. Dash, N. Tombros and B. J. V. Wees, Appl. Phys. Lett., 2011, 99, 1643.

171 J. A. Leon, N. C. Mamani, A. Rahim, L. E. Gomez, M. A. P. D. Silva and G. M. Gusev, Graphene, 2014, 03, 2535.

172 M. Wang, S. K. Jang, W. J. Jang, M. Kim, S. Y. Park, S. W. Kim, S. J. Kahng, J. Y. Choi, R. S. Ruoff and Y. J. Song, Adv. Mater., 2013, 25, 2746-2752.

173 M. Wang, M. Kim, D. Odkhuu, J. Lee, W. J. Jang, S. J. Kahng, N. Park, R. S. Ruoff, Y. J. Song and S. Lee, ACS Nano, 2014, 8, 5478-5483.

174 S. M. Kim, A. Hsu, P. T. Araujo, Y. H. Lee, T. Palacios, M. Dresselhaus, J. C. Idrobo, K. K. Kim and J. Kong, Nano Letters, 2013, 13, 933-941.

175 T. Gao, X. Song, H. Du, Y. Nie, Y. Chen, Q. Ji, J. Sun, Y. Yang, Y. Zhang and Z. Liu, Nat. Commun., 2011, 6, 6835.

176 W. Gannett, W. Regan, K. Watanabe, T. Taniguchi, M. F. Crommie and A. Zettl, Appl. Phys. Lett., 2011, 98, 2421051-2421053.

177 Z. Liu, L. Song, S. Zhao, J. Huang, L. Ma, J. Zhang, J. Lou and P. M. Ajayan, Nano Lett., 2011, 11, 2032-2037.

178 Z. Yan, Z. Peng, Z. Sun, J. Yao, Y. Zhu, Z. Liu, P. M. Ajayan and J. M. Tour, ACS Nano, 2011, 5, 8187-8192.

179 S. Tang, G. Ding, X. Xie, J. Chen, C. Wang, X. Ding, F. Huang, W. Lu and M. Jiang, Carbon, 2012, 50, 329-331.

180 S. Tang, H. Wang, a. Y. Zhang, A. Li, H. Xie, X. Liu, L. Liu, T. Li, F. Huang and X. Xie, Sci. Rep., 2013, 3, 2666.

181 X. Song, G. Teng, Y. Nie, J. Zhuang, J. Sun, D. Ma, J. Shi, Y. Lin, D. Feng and Y. Zhang, Nano Lett., 2016, 16, 61096116.

182 L. Britnell, R. V. Gorbachev, R. Jalil, B. D. Belle, F. Schedin, A. Mishchenko, T. Georgiou, M. I. Katsnelson, L. Eaves and S. V. Morozov, Science, 2011, 335, 947-950.

183 C. Dean, A. F. Young, L. Wang, I. Meric, G. H. Lee, K. Watanabe, T. Taniguchi, K. Shepard, P. Kim and J. Hone, Solid State Commun., 2012, 152, 1275-1282.

184 L. A. Ponomarenko, R. V. Gorbachev, G. L. Yu, D. C. Elias, R. Jalil, A. A. Patel, A. Mishchenko, A. S. Mayorov, C. R. Woods and J. R. Wallbank, Nature, 2013, 497, 594.

185 C. R. Dean, L. Wang, P. Maher, C. Forsythe, F. Ghahari, Y. Gao, J. Katoch, M. Ishigami, P. Moon and M. Koshino, Nature, 2013, 497, 598.

186 B. Hunt, J. D. Sanchezyamagishi, A. F. Young, M. Yankowitz, B. J. Leroy, K. Watanabe, T. Taniguchi, P. Moon, M. Koshino and P. Jarilloherrero, Science, 2013, 340, 1427.

187 W. Yang, G. Chen, Z. Shi, C. C. Liu, L. Zhang, G. Xie, M. Cheng, D. Wang, R. Yang and D. Shi, Nat. Mater., 2013, 12, 792-797.

188 C. Zhang, Nat. Commun., 2015, 6, 6519.

189 K. Watanabe, T. Taniguchi, T. Kuroda and H. Kanda, Diamond Relat. Mater., 2006, 15, 1891-1893.

190 K. Watanabe, T. Taniguchi, T. Kuroda and H. Kanda, Appl. Phys. Lett., 2006, 89, 141902-141903. 
191 J. Wang, S. Cao, P. Sun, Y. Ding, Y. Li and F. Ma, RSC Adv., 2016, 6, 111345-111349.

192 X. Yang, F. Zhai, H. Hu, D. Hu, R. Liu, S. Zhang, M. Sun, Z. Sun, J. Chen and Q. Dai, Adv. Mater., 2016, 28, 2931.

193 A. Pakdel, C. Zhi, Y. Bando and D. Golberg, Mater. Today, 2012, 15, 256-265.

194 A. S. Mayorov, R. V. Gorbachev, S. V. Morozov, L. Britnell, R. Jalil, L. A. Ponomarenko, P. Blake, K. S. Novoselov, K. Watanabe and T. Taniguchi, Nano Lett., 2011, 11, 2396.

195 J. Xue, J. Sanchez-Yamagishi, D. Bulmash, P. Jacquod, A. Deshpande, K. Watanabe, T. Taniguchi, P. JarilloHerrero and B. J. Leroy, Nat. Mater., 2011, 10, 282-285.

196 M. Yankowitz, Nat. Phys., 2012, 8, 382-386.

197 F. Hüser, T. Olsen and K. S. Thygesen, J. Child Health Care, 2013, 61, 503-511.

198 E. Wang, X. Lu, S. Ding, W. Yao, M. Yan, G. Wan, K. Deng, S. Wang, G. Chen and L. Ma, Nat. Phys., 2016, 12, 11111115.

199 S. Okada and A. Oshiyama, Phys. Rev. Lett., 2001, 87, 146803.

200 C. Jin, Y. H. Kim, K. J. Chang and D. Tománek, Phys. Rev. B: Condens. Matter Mater. Phys., 2003, 67, 181-183.

201 A. Ramasubramaniam and D. Naveh, Phys. Rev. B: Condens. Matter Mater. Phys., 2011, 84, 173-177.
202 N. Berseneva, A. V. Krasheninnikov and R. M. Nieminen, Phys. Rev. Lett., 2011, 107, 035501.

203 X. Ding, H. Sun, X. Xie, H. Ren, F. Huang and M. Jiang, Phys. Rev. B, 2011, 84, 4327-4331.

204 C. R. Dean, A. F. Young, I. Meric, C. Lee, L. Wang, S. Sorgenfrei, K. Watanabe, T. Taniguchi, P. Kim and K. L. Shepard, Nat. Nanotechnol., 2010, 5, 722-726.

205 K. H. Lee, H. J. Shin, J. Lee, I. Y. Lee, G. H. Kim, J. Y. Choi and S. W. Kim, Nano Lett., 2012, 12, 714.

206 J. Lee, T. J. Ha, K. N. Parrish and S. F. Chowdhury, IEEE Electron Device Lett., 2013, 34, 172-174.

207 M. Iqbal, M. Iqbal, X. Jin, J. Eom and C. Hwang, J. Mater. Chem. C, 2014, 2, 7776-7784.

208 C. C. Chen, Z. Li, L. Shi and S. B. Cronin, Appl. Phys. Lett., 2014, 104, 081908.

209 C. Chun-Chung, Zhen and S. Cronin, Nano Res., 2015, 8, 666-672.

210 F. Withers, P. Z. O. Del, S. Schwarz, S. Dufferwiel, P. Walker, T. Godde, A. P. Rooney, A. Gholinia, C. Woods and P. Blake, Nano Lett., 2015, 15, 8223-8228.

211 X. Wang and F. Xia, Nat. Mater., 2015, 14, 264-265.

212 X. Li, S. Lin, X. Lin, Z. Xu, P. Wang, S. Zhang, H. Zhong, W. Xu, Z. Wu and F. Wei, Opt. Express, 2016, 24, 134.

213 Y. Xu, Z. Guo, H. Chen, Y. Yuan, J. Lou, X. Lin, H. Gao, H. Chen and B. Yu, Appl. Phys. Lett., 2011, 99, 133109. 